Proceedings of the

International Geometry Center

Vol. 11, no. 2 (2018) pp. 16-47

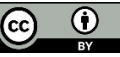

\title{
On measures of nonplanarity of cubic graphs
}

\author{
Leonid Plachta
}

\begin{abstract}
We study two measures of nonplanarity of cubic graphs $G$, the genus $\gamma(G)$, and the edge deletion number $e d(G)$. For cubic graphs of small orders these parameters are compared with another measure of nonplanarity, the rectilinear crossing number $\overline{c r}(G)$. We introduce operations of connected sum, specified for cubic graphs $G$, and show that under certain conditions the parameters $\gamma(G)$ and $e d(G)$ are additive (subadditive) with respect to them.

The minimal genus graphs (i.e. the cubic graphs of minimum order with given value of genus $\gamma$ ) and the minimal edge deletion graphs (i.e. cubic graphs of minimum order with given value of edge deletion number $e d$ ) are introduced and studied. We provide upper bounds for the order of minimal genus and minimal edge deletion graphs.
\end{abstract}

Анотація. 3 відомої теореми Куратовського випливає, що кубічний граф є непланарним тоді і тільки тоді, коли він не місить підграфів, гомеоморфних $K_{3,3}$. Для непланарних графів існує декілька характеристик графа, які визначають міру його непланарності. Для заданого 3 -зв'язного кубічного графу $G$ позначимо через $\operatorname{ed}(G)$ найменше число ребер в $G$, після викидання яких дістанемо планарний підграф, а через $g(G)$ (орієнтовний) рід графа $G$. Крім того, нехай $\overline{c r}(G)$ позначає мінімальне число внутрішніх перетинів ребер графа $G$ серед усіх прямолінійних імерсій графа в площині. Кубічний граф $G$ називається $k$ мінімальним відносно параметра $e d(G)$ (відповідно, параметрів $\overline{c r}(G)$, $g(G)$ ), якщо $\operatorname{ed}(G)=k$ (відповідно, $\overline{c r}(G)=k, g(G)=k$ ) і порядок графа $G$ є мінімальним серед усіх 3-зв'язних кубічних графів з даною властивістю.

В роботі досліджуються $k$-мінімальні відносно параметрів $е d(G)$ і $g(G)$ 3-зв'язних кубічних графи. Описані операції на 2-зв'язних і 3-зв'язних кубічних графах (зв'язна сума, подвійна зв'язна сума), які мають властивість адитивності (субадитивності) відносно параметрів $е d(G)$ і $g(G)$.

The research partially supported by the Polish Ministry of Science and Higher Education 2010 Mathematics Subject Classification: 05C10, 05C15

Keywords: nonplanar graphs, cubic graphs, genus, edge deletion number, connected sum of graphs, minimal graphs 
Обчислені характеристики $e d(G)$ і $g(G) k$-мінімальних відносно параметра $\overline{c r}(G)$ кубічних графів для малих чисел $k$. Дається порівняння характеристик $e d(G), g(G)$ і $\overline{c r}(G)$ для зв'язних кубічних графів $G$.

Запропонований конструктивний метод, який дозволяє отримувати 2зв'язні і циклічно 4-зв'язні кубічні графи $G$ з як завгодно великими характеристиками $\operatorname{ed}(G)$ i $g(G)$. Даний метод базується на адитивних властивостях операцій "зв'язна сума" і “подвійна зв'язна сума" на кубічних графах. Отримані також верхні оцінки порядку мінімальних відносно характеристик $e d(G)$ і $g(G)$ графів $G$, в класі 2-зв'язних і 3-зв'язних кубічних графів $G$. Сформульована також відкрита проблема, яка стосується нижньої оцінки для порядку $k$-мінімальних відносно параметрів $e d(G)$ і $g(G)$ кубічних графів.

\section{INTRODUCTION}

We consider finite graphs $G=(V, E)$ without loops and multiple edges. The number of vertices of $G$ is called the order of $G$ and denoted by $|G|$. The number of edges $|E|$ of the graph $G$ is called its size and denoted by $\|G\|$. The Kuratowski theorem states that a graph $G$ is planar if and only if it does not contain subgraphs homeomorphic to $K_{5}$ and $K_{3,3}$. A regular graph of valence 3 is called cubic. For cubic graphs, the only forbidden graphs are those which are not homeomorphic to $K_{3,3}$. There are different measures of nonplanarity of a graph. Let us recall their definitions.

For a given connected graph $G$ denote by $\gamma(G)$ the (orientable) genus of $G$ i.e. the minimal genus of an orientable closed connected surface $M$ such that $G$ has an embedding in $M$. Note that each such embedding is 2-cell. The problem of deciding whether a cubic graph $G$ has the genus $\gamma(G) \leq m$ is known to be NP-complete, [21]. There are some upper and lower bounds of $\gamma(G)$ for different classes of graphs $G$, [19]. For cubic graphs $G$, the precise values of the parameter $\gamma(G)$ are known only for special classes of them (for example, for some snarks, etc., see [15, 19]).

Another well known measure of nonplanarity of a graph $G$ is the crossing number $\operatorname{cr}(G)$ (the rectilinear crossing number $\overline{c r}(G)$ ). This is the minimal number of proper double crossings of edges among all immersions of $G$ in the plane (the minimal number of proper double crossings of edges among all rectilinear immersions of $G$ in the plane, respectively). The computation of the crossing number of a graph is also an NP-complete problem, [7]. Note that, in general, $\operatorname{cr}(G)$ and $\overline{c r}(G)$ are distinct numbers, [2]. There are estimations of the parameters $\operatorname{cr}(G)$ and $\overline{c r}(G)$ for complete graphs, complete bipartite graphs, and other special classes of graphs (see, for example $[10,20])$. The precise values of $\operatorname{cr}(G)$ and $\overline{c r}(G)$ are known only for particular nonplanar graphs (for example, for small complete and complete bipartite graphs, $[17,20])$. 
For a given graph $G$, denote by $e d(G)$ the minimal number of edges in $G$ such that after their deletion the resulting graph becomes planar. The parameter ed $(G)$ is called the edge deletion number of $G$ and the corresponding problem of finding the minimal set of edges to be deleted in a graph $G$ is known as MINED. Even for cubic graphs, the problem MINED is known to be NP-complete, [5]. Algorithms of computing ed $(G)$, in particular, for cubic graphs, are described in $[3,5,4]$.

Comparing with the parameters $\gamma(G)$ and $\operatorname{cr}(G)$, there are much more fewer results concerning evaluation of the number ed $(G)$. Pegg jr and Exoo [12] introduced the notion of a minimal crossing graph. For a given natural number $k$ a cubic graph $G$ is called minimal $k$-crossing graph (in original, $k$-crossing graph [12]) if $G$ has a minimal order among all cubic graph $H$ with $\overline{c r}(H)=k$. By this analogy, we introduce minimal $k$-genus and minimal $k$-edge deletion graphs. Denote by $\beta(G)$ the cyclomatic number of the connected graph $G, \kappa(G)$ the vertex connectivity and $\lambda(G)$ the edge connectivity of $G$. For graphs $G$ of maximal degree at most three the number $\lambda(G)$ and $\kappa(G)$ coincide, [6]. By this reason, for cubic graphs $G$ we will abbreviate the terminology and use the term "the connectivity of $G$ ".

We say that a connected graph $G=(V, E)$ is cyclically $k$-edge connected if no set of fewer than $k$ edges is cycle-separating in $G$. The edge cyclic connectivity $\zeta(G)$ of the cubic graph $G$ is the largest integer $k \leq \beta(G)$ for which $G$ is cyclically $k$-edge connected. For any cubic connected graph $G$ we have obviously $\kappa(G)=\lambda(G) \leq \zeta(G)$. Note that $\zeta(G)$ is equal to $\beta(G)$ if and only if $G$ does not have any cycle-separating edge cut. Moreover for cubic graphs $G$ with $\zeta(G) \leq 3$ the values of vertex connectivity, edge connectivity and cyclic $k$-edge connectivity coincide, [16]. As an example, for the Petersen graph $P$ we have $\kappa(P)=\lambda(P)=3$ but $\zeta(P)=5$.

We say that a connected cubic graph $G$ is cyclically $k$-vertex connected if it contains no cycle-separating vertex cut with fewer than $k$ vertices. For exception of few graphs (which are $K_{4}, K_{3,3}$ and the multigraph $\Theta_{2}$ ), the notions of cyclically $k$-vertex connected graph and cyclically $k$-edge connected graph coincide, [16, Proposition 3].

In Section 2, we evaluate genus and edge deletion number of minimal $k$-crossing graphs for small numbers $k$. These auxiliary results are used in Section 3.

Battle et al. [1] have shown that the genus of any connected graph is equal to the sum of blocks with respect to its block decomposition. This is perhaps the first known result on additivity of the (orientable) genus of graph. The operation of the vertex amalgamation applied to 2-connected cubic graphs gives a separable graph which contains a vertex of degree 4 . 
Another operation is the edge amalgamation of graphs $G_{1}$ and $G_{2}$, [13]. Miller, [13], introduced the generalized genus of a graph and showed that it is additive with respect to the edge amalgamation of two graphs. The operation of edge amalgamation does not preserve the class of cubic graphs. In [8], Gross also studied bar-amalgamation of graphs. All these operations, when applied to cubic graphs, produce the graphs which are outside the given class. Moreover they are not compatible with such properties of graphs (including cubic graphs) as the vertex connectivity and the edge connectivity. Therefore they cannot serve as a good tool for the construction of graphs with big numbers of the parameters $e d$ and $\gamma$ inside the class of cubic graphs with the given connectivity.

In Section 3, we introduce two operations of connected sum which are suitable for cubic graphs. We study additivity properties of genus and edge deletion number with respect to these operations. The first operation, when applied to two 2-connected cubic graphs, results in a 2-connected cubic graph. Similarly, the second operation preserves, in general, the class of 3-connected (or even cyclically 4-edge connected) cubic graphs. Additivity properties of cubic graphs are provided by Theorems 3.1, 3.2 and 3.3 (subject to the parameter $\gamma$ ), and by Theorems 3.4 and 3.5 (subject to the parameter ed). By using using these properties, we provide upper bounds for the order of a 2-connected and 3-connected cubic graphs $G$, which are minimal with respect to these parameters (Corollaries 3.1-3.4). These are the main results of the paper.

In [18] we use more subtle arguments for obtaining upper bounds of the order of minimal cubic graphs $G$ with prescribed value of the parameter $\gamma$. The additivity properties of the parameter $\gamma$ given by Theorems 3.1, 3.2 and 3.3 are also essential in this relation.

\section{Measures of NONPLANARITy OF CUBIC GRAPHS: SMALL ORDERS}

We start by considering the parameters $\operatorname{cr}(G)$ and $\overline{c r}(G)$ for small cubic graphs $G$ and compare these numbers with the parameters $\gamma(G)$ and $e d(G)$. Denote by $g(G)$ the girth of the graph $G$. In our study of the (orientable) genus of cubic graphs we shall use the notion of the rotation system on a graph.

A rotation system on a graph $G=(V, E)$ is a family $\Pi=\left\{\pi_{v}: v \in V\right\}$, where $\pi_{v}$ is a cyclic permutation of the edges incident with $v$. With any 2-cell embedding $\varphi$ of a graph $G$ into an oriented closed surface $S$ it is associated a rotation system $\Pi$ on $G$. The pair $(G, \Pi)$ is called a rotation graph. Moreover for a given rotation graph $(G, \Pi)$ one can construct a system $R$ of (oriented) circuits on $G$ in such a way that each edge $e$ of $G$ is contained twice in the circuits, but with opposite orientations. The circuits 
$c$ from $R$ can be thought of as oriented boundaries of 2-dimensional discs $D_{c}$. Gluing together the family of discs $D_{c}, c \in R$, along their oriented boundaries, we shall obtain an oriented closed surface $S$ and this provides a natural 2-cell embedding $\psi$ of $G$ into $S$. The circuits $c$ from $R$ are called the facial cycles of the embedding $\psi$. The correspondence between the 2cell embeddings of a graph $G$ into oriented surfaces and rotation systems on $G$ is one-to-one, in a usual sense. For more details see [9], [14].

It is easy to see that we have the following inequalities:

$$
\gamma(G) \leq e d(G) \leq \operatorname{cr}(G) \leq \overline{c r}(G) .
$$

It can be shown that for cubic graphs the difference between any two of the parameters $\gamma(G), e d(G), \operatorname{cr}(G)$ of $G$ can be arbitrarily large. This can be proven, for example, by using results of Sections 2 and 3. Moreover, there exist graphs $G$ for which the number $\operatorname{cr}(G)$ is less than $\overline{c r}(G)$ (more precisely, $\operatorname{cr}(G)=4$ and $\overline{c r}(G)=m$ for any $m>4$, [2]).

We shall say that a cubic graph $G$ is minimal l-genus graph if $\gamma(G)=l$ and it is of minimum order among all 2-connected cubic graphs with this property. Similarly, for a given nonnegative integer $l$, a cubic graph $G$ is minimal $l$-edge deletion graph, if $\operatorname{ed}(G)=l$ and $G$ is of minimum order among all 2-connected cubic graphs with this property.

In this section, we evaluate or estimate the order of minimal graphs with respect to parameters $\gamma$ and $e d$ for small numbers $l$. First count all minimal $l$-crossing graphs $G$ for small values $l$. Minimal $l$-crossing graphs have been described up to value $l \leq 8$ in [12]. Note that for $l=9$ it is unknown any minimal crossing graph $G$. At present, for $l \geq 10$, there are known only hypothetically minimal $l$-crossing graphs. Using minimal $l$-crossing graphs, we will find some minimal cubic graphs with respect to parameters ed and $\gamma$. For cubic graphs of small order we use the notations as in [12].

In the following, we will work in the piece wise linear category PL, [11]. Therefore surfaces are 2-dimensional PL-manifolds, graphs are 1dimensional polyhedra, the maps (embeddings) of graphs are PL-maps (PL-embeddings) and the images of graphs under such maps are subpolyhedra of PL-manifolds (surfaces). For more detailed information about the category PL see also [14].

1. For $l=1$ there is a unique minimal crossing graph, the graph $K_{3,3}$. We have obviously

$$
\operatorname{ed}\left(K_{3,3}\right)=\overline{c r}\left(K_{3,3}\right)=\operatorname{cr}\left(K_{3,3}\right)=\gamma\left(K_{3,3}\right)=1 .
$$

2. For $l=2$ there are two minimal crossing graphs. These are the Petersen graph $P$ (see Figure 2.1b) and the graph $C N G 2 B$ (see Figure 2.1a). We have obviously

$$
\operatorname{ed}(P)=2, \quad \gamma(P)=1, \quad \operatorname{ed}(C N G 2 B)=\gamma(C N G 2 B)=1 .
$$




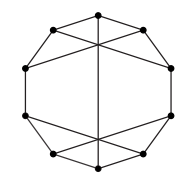

a)

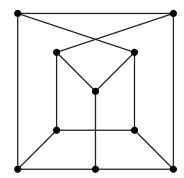

b)

Figure 2.1. The minimal 2-crossing graphs

We shall say that a system $F$ of (oriented) circuits in a graph $G$ is admissible if it determines a rotation system or can be completed to a rotation system on $G$ by adding some additional circuits in $G$. In the latter case, we will say that $F$ is incomplete. Note that for a connected cubic graph $G$ of order 12, any incomplete admissible system $F$ on $G$ consisting of 4 circuits can be completed to a rotation system $R$ that induces embedding of $G$ in torus or the sphere $S^{2}$.

Lemma 2.1. For any connected cubic graph $G$ of order 12 we have

$$
\gamma(G) \leq 1
$$

Proof. Let $G$ be a connected cubic graph of order 12. We know from [12] that if $|G| \leq 12$, then $\overline{c r}(G) \leq 2$. If $\kappa(G)=1$, the assertion follows (by using for example results from [1]). If $\kappa(G)=2$, then after removal two nonincident edges from $G$ we shall obtain two planar subcubic graphs, $G_{1}$ and $G_{2}$. The only case, when one can worry about, is that $G_{1}$ and $G_{2}$ are isomorphic to $K_{3,3}-e$ where $e$ is an edge of $K_{3,3}$. But in this case, $G$ is isomorphic to a connected sum of two copies of $K_{3,3}$ and has genus 1 (see Section 3). Therefore we may assume that $G$ is 3-connected. If $\overline{c r}(G)=1$ we have obviously $\gamma(G)=1$. If $\overline{c r}(G)=2$ and the equality reaches via a straight line drawing $G$ in the plane, in which one edge intersects two another edges, the assertion also easily follows.

Assume that there is an immersion of $G$ in the oriented plane $P$ in which we have crossings of two pairs of different edges: $e_{1}$ and $e_{2}$, and $f_{1}$ and $f_{2}$. Deleting $e_{1}, e_{2}, f_{1}$ and $f_{2}$ from $G$, we get a subcubic multigraph $H$ which has a natural embedding $\varphi$ in $P$. We may assume without loss of generality that $H$ is connected, otherwise one can use a flip and redraw $G$ in the plane with a fewer number of crossings. Denote by $\Pi$ the rotation system on $H$ associated with the embedding $\varphi$. Now consider all possible configurations of the induced plane embedding of the (multi)graph $H$ and the positions of the deleted edges with respect to it.

a) There is an inner face $r$ of the embedding $\varphi$ which contains two pairs of crossing edges, $e_{1}$ and $e_{2}$, and $f_{1}$ and $f_{2}$. We have three types of configurations describing positions of these edges inside a regular face $r$. In 
any case, we are able to define an admissible system of circuits in $G$ which, after a suitable completion, generates an embedding of $G$ into torus.

In the first case (see Figure 2.2), we have inside $r$ two internal vertices of $G$ and two crossings of different pairs of edges. The position of external two edges of $H$ and incident vertices is irrelevant and so, is not indicated here. One can replace the facial circuit $d r$ of the rotation system $\Pi$ with three new circuits $c_{1}, c_{2}, c_{3}$ as indicated in Figure 2.2. The system of circuits $F=\left\{c_{1}, c_{2}, c_{3}\right\}$ in $G$ is admissible and contain each "internal" edge of the immersed graph $G$ twice, but with opposite orientations. The orientation of edges positioned on $d r$ coincide with the one of the cycle $d r$. We can complete $F$ to a rotation system on $G$ by adding three outer circuits of $\Pi$, $u_{1}, u_{2}$ and $u_{3}$. The system of circuits $\mathcal{F}=\left\{c_{1}, c_{2}, c_{3}, u_{1}, u_{2}, u_{3}\right\}$ defines a rotation system $T$ on $G$ of genus 1 .

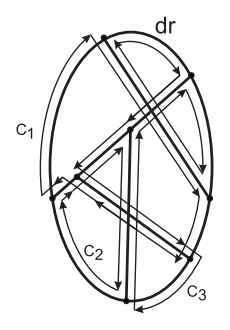

FiguRe 2.2.

In the second case, we have inside $r$ one internal vertex of $G$ and two crossings of edges, as shown in Figure 2.3a). The edges of $G$ positioned inside $r$ are called internal while the edges in the exterior of $r$ are called external (subject to the given immersion of $G$ in $P$ ). Let $E^{\prime}$ be the set of external edges of $G$. The vertices of $G$ incident to external edges are called external. Denote by $H_{1}$ the graph $G-E^{\prime}$. Then $H_{1}$ is immersed via $\varphi$ in the closure of the face $r$. Consider in $G$ the system of circuits

$$
F_{1}=\left\{c_{1}, c_{2}, c_{3}, c_{4}, c_{5}, c_{6}\right\}
$$

indicated in Figure 2.3b). $F_{1}$ defines an embedding of $H_{1}$ into a sphere $S^{2}$. We have two possibilities:

a) $G$ is obtained from $H_{1}$ by gluing two nonincident edges $e_{1}$ and $e_{2}$ to $H_{1}$;

b) $G$ is obtained from $H_{1}$ by gluing the graph $K_{1,3}$ along three vertices of degree 1 .

Consider the subcase a). Suppose that the end vertices of $e_{1}$ (or $\left.e_{2}\right)$ are lying on the same circuit $c_{i}$. One can replace $c_{i}$ in the system $F_{1}$ with two new circuits, $c_{i}^{\prime}$ and $c_{i}^{\prime \prime}$, as shown in Figure 2.3c). Denote by $F_{2}$ the new 


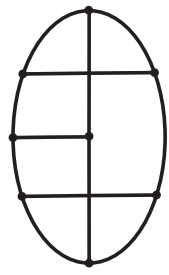

a)

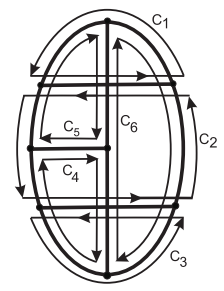

b)

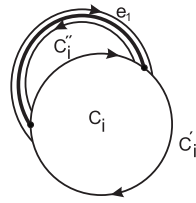

C)

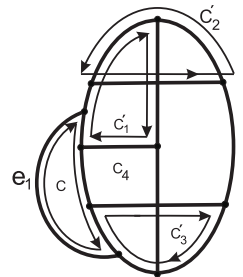

d)

FIGURE 2.3.

family consisting of seven circuits. Obviously $F_{2}$ is a rotation system on the graph $H_{1} \cup e_{1}\left(H_{1} \cup e_{2}\right.$, respectively). The remaining edge $e_{k}$ from $\left\{e_{1}, e_{2}\right\}$ joins the vertices of different circuits of $F_{2}$, say $d_{i}$ and $d_{j}$. This results in a single circuit $d$ in $G$ containing both $d_{1}$ and $d_{2}$ and the edge $e_{k}$, which is involved twice and with opposite directions. Finally we get a rotation system $R$ on $G$ consisting of six circuits.

Now suppose that both $e_{1}$ and $e_{2}$ joins the vertices of the same circuits, $c_{i}$ and $c_{j}$ of $F_{1}$. The vertices of $e_{1}$ and $e_{2}$ divide $c_{i}$ into two directed paths, $l_{1}$ and $l_{2}$, and $c_{j}$ into two directed paths, $m_{1}$ and $m_{2}$. Combining the paths $l_{1}, l_{2}, m_{1}$ and $m_{2}$ with the oriented edges $e_{1}$ and $e_{2}$, we get two circuits in $G, d_{i}$ and $d_{j}$. Each edge $e_{i}, i=1,2$, is contained both in $d_{i}$ and $d_{j}$, but with opposite orientation. Replacing the pair of circuits $c_{i}$ and $c_{j}$ with the pair $d_{i}$ and $d_{j}$, we obtain an admissible system $F$ in $G$, consisting of six circuits. Therefore $F$ defines in $G$ a rotation system of genus one.

In the remaining cases, we can take three circles $c_{1}^{\prime}, c_{2}^{\prime}$ and $c_{3}^{\prime}$ of $F_{1}$, endow them with a suitable orientation, and add to them a new circuit $c$ of $G$, which contains $e_{1}$ or $e_{2}$. This results in an admissible system $F^{\prime}$ on $G$ consisting of 4 circuits. The choice of $c_{1}^{\prime}, c_{2}^{\prime}$ and $c_{3}^{\prime}$ depends on a position of vertices of $e_{1}$ or $e_{2}$ on $d r$ (see, for example, Figure 2.3d). Completing $F^{\prime}$ by two new circuits, we get a rotation system $R$ on $G$ of genus one.

The subcase b) is handled in the same way as subcase a). We omit here the details.

The third case of configuration for $H_{1}$ is indicated in Figure 2.4a). We also have two possibilities:

a) $G$ is obtained from $H_{1}$ by gluing up two nonincident edges $e_{1}$ and $e_{2}$;

b) $G$ is obtained from $H_{1}$ by gluing up the graph $K_{1,3}$ along three vertices of degree 1.

There is no essential difference between the subcases a) and b). Let us consider the subcase a). We can choose two circuits $c_{1}$ and $c_{2}$ in $H_{1}$ and add to them two circuits $d_{1}$ and $d_{2}$ in $G$ such that $d_{1}$ contains the edge $e_{1}$ 
and $d_{2}$ contains the edge $e_{2}$. As a result, we get an incomplete admissible system $F^{\prime}$ of circuits in $G$. The choice of circuits $c_{1}$ and $c_{2}$ depends on the position of vertices of the edges $e_{1}$ and $e_{2}$ on $d r$, see Figure 2.4b) and c). Then $F^{\prime}$ can be completed to an admissible system $F$ in $G$, consisting of six circuits. An exceptional case of the pairs of crossing edges $e_{1}, e_{2}$ and $f_{1}, f_{2}$

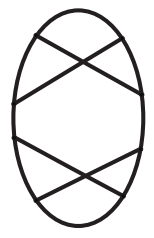

a)

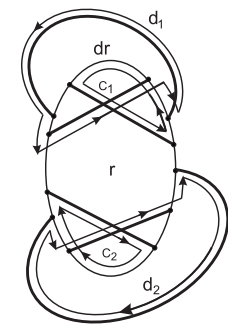

b)

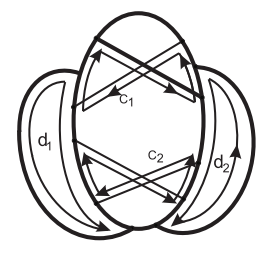

c)

FiguRE 2.4.

inside a nonregular face $r$ is shown in Figure 2.5. The following family of circuits in $G$ determines a rotation system $R$ of genus one:

$$
\begin{array}{ll}
c_{1}=\left(v_{1}, v_{2}, v_{3}, v_{4}\right), & c_{2}=\left(v_{4}, v_{3}, v_{5}, v_{6}, u_{6}\right), \\
c_{3}=\left(v_{6}, u_{2}, u_{3}, u_{5}, u_{6}\right), & c_{4}=\left(u_{2}, u_{1}, u_{4}, u_{3}\right), \\
c_{5}=\left(u_{1}, u_{2}, v_{6}, v_{5}, v_{1}, v_{4}, u_{6}, u_{5}\right), & c_{6}=\left(u_{1}, u_{5}, u_{3}, u_{4}, v_{2}, v_{1}, v_{5}, v_{3}, v_{2}, u_{4}\right) .
\end{array}
$$

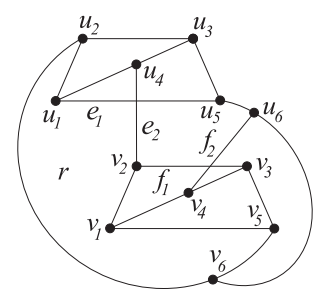

Figure 2.5.

b) There are two faces $r_{1}$ and $r_{2}$ of the embedding $\varphi$ such that $r_{1}$ contains the crossing of $e_{1}$ and $e_{2}$, and $r_{2}$ contains the crossing of $f_{1}$ and $f_{2}$.

If $r_{1}$ and $r_{2}$ are disjoint, the existence of a rotation system $\Pi^{\prime}$ on $G$ with 6 circuits is obvious. If $r_{1}$ and $r_{2}$ have a unique edge in common, we have a configuration shown in Figure 2.6. There is a rotation system $R$ on $G$ with 6 facial circuits. We indicate here only a noncomplete admissible system consisting of four circuits, $F=\left\{c_{1}, c_{2}, c_{3}, c_{4}\right\}$. The circuits are the following:

$$
c_{1}=\left(u_{2}, u_{3}, u_{4}, u_{1}\right), \quad c_{2}=\left(u_{1}, u_{4}, u_{5}, u_{11}, u_{12}\right),
$$




$$
c_{3}=\left(u_{11}, u_{5}, u_{6}, u_{9}, u_{10}\right), \quad c_{4}=\left(u_{9}, u_{6}, u_{7}, u_{8}\right)
$$

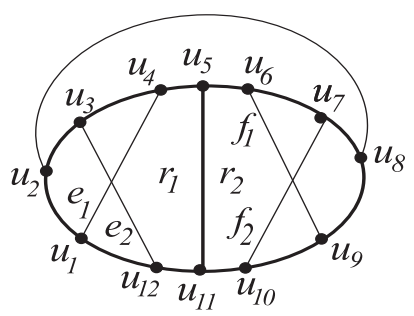

FiguRE 2.6.

Assume now that $r_{1}$ and $r_{2}$ have two edges in common, see Figure 2.7. In this case, we indicate a rotation system $R$ on $G$ with the following six circuits:

$$
\begin{array}{ll}
c_{1}=\left(u_{2}, u_{1}, v_{2}, v_{1}\right), & c_{2}=\left(v_{5}, v_{4}, u_{5}, u_{4}\right), \\
c_{3}=\left(u_{1}, u_{2}, u_{3}, u_{4}, u_{5}, u_{6}\right), & c_{4}=\left(v_{1}, v_{2}, v_{3}, v_{4}, v_{5}, v_{6}\right), \\
c_{5}=\left(u_{2}, v_{1}, v_{6}, u_{6}, u_{5}, v_{4}, v_{3}, u_{3}\right), & c_{6}=\left(u_{1}, u_{6}, v_{6}, v_{5}, u_{4}, u_{3}, v_{3}, v_{2}\right) .
\end{array}
$$

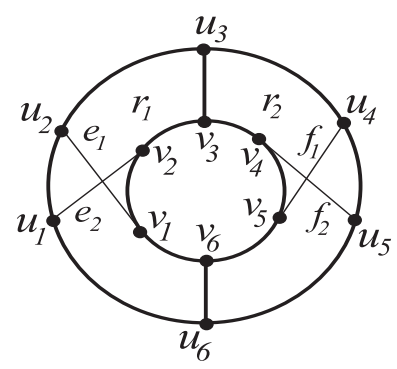

FiguRE 2.7.

c) It can occur that $H$ is a multigraph with three loops and the pairs of crossing edges of $G$ are situated in the outer face $p$ of the embedding $\varphi$. We depict in Figure 2.8 such a configuration. In this case, we indicate the following noncomplete admissible system of circuits in $G$ :

$$
\begin{array}{ll}
c_{1}=\left(u_{10}, u_{9}, u_{12}, u_{11}\right), & c_{2}=\left(u_{11}, u_{12}, u_{3}, u_{4}\right), \\
c_{3}=\left(u_{4}, u_{3}, u_{2}, u_{1}\right), & c_{4}=\left(u_{5}, u_{6}, u_{7}\right) .
\end{array}
$$

Note that the case when one pair of crossing edges of $G$ is inside $p$ and the other one is inside a region bounded by a loop of $H$ is not admissible by the assumption that the graph $G$ is 2-connected. Lemma 2.1 is completed. 


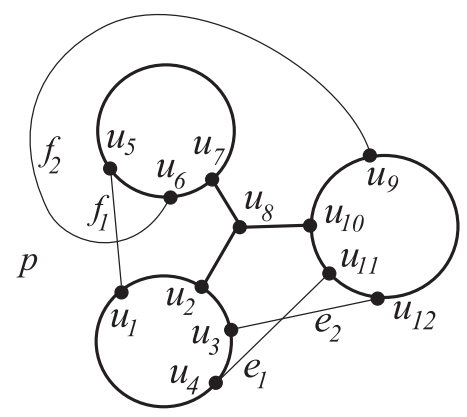

Figure 2.8 .

3. For $l=3$ there are eight minimal crossing graphs. We count them according to [12]: $C N G 3 A, C N G 3 B, C N G 3 D, C N G 3 E, C N G 3 F$, $C N G 3 H, G P(7,2)$, the Heawood graph $H$ (see Figure 2.9).

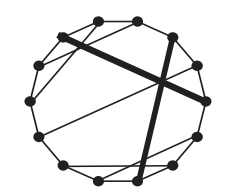

CNG 3A

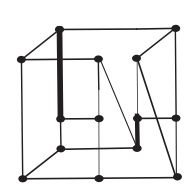

CNG 3E

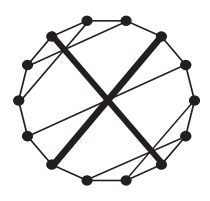

CNG 3B

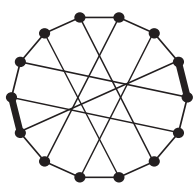

CNG $3 \mathrm{~F}$

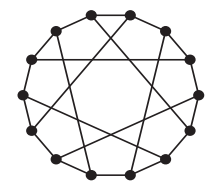

Heawood graph

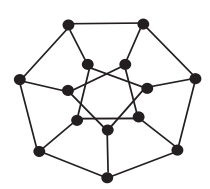

$\operatorname{GP}(7,2)$

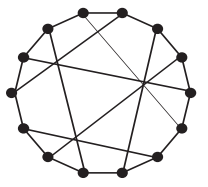

CNG 3D

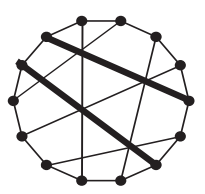

$\mathrm{CNG} 3 \mathrm{H}$

FiguRE 2.9. The minimal 3-crossing graphs

By direct computation, we have

$$
\begin{aligned}
e d(C N G 3 A) & =e d(C N G 3 B)=\operatorname{ed}(C N G 3 E) \\
& =\operatorname{ed}(C N G 3 F)=\operatorname{ed}(C N G 3 H)=2 .
\end{aligned}
$$

In Figure 2.9, for each of these graphs we indicate by bold line the two edges after removal of which we obtain a planar subgraph. In Figure 2.10a), we indicate 3 -crossing drawing of the graph $G P(7,2)$. By bold lines there are indicated two edges in $G P(7,2)$ after removal of which we obtain a planar subgraph. Similarly, in Figure 2.10b) we indicate 3-crossing drawing of the graph $C N G 3 D$. Removing two bold edges from it also leads to a planar subgraph. 


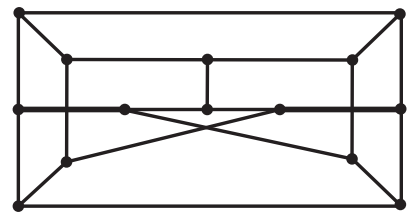

a) $G P(7,2)$

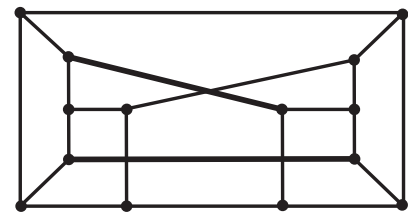

b) $C N G 3 D$

FiguRE 2.10.

Lemma 2.2. For the Heawood graph $H$ we have ed $(H)=3$.

Proof. It is well known that $H$ is symmetric graph. Remove any edge $e$ from $H$. The resulting graph $U$ can be represented as a sum of two subgraphs $H_{1}$ and $H_{2}$ and the edge $f$, see Figure 2.11. The subgraphs $H_{1}$ and $H_{2}$ have in common a path $l$ of length 5 . Denote by $E(l)$ the set of edges of the path $l$.

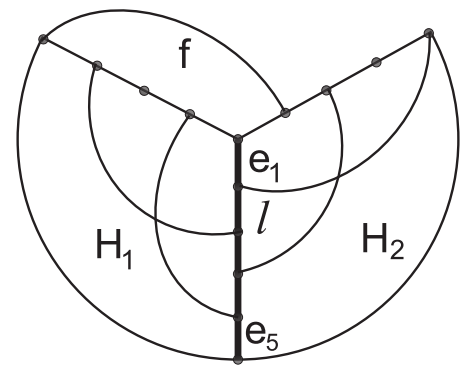

FIGURE 2.11.

Removing the edge $f$ from $U$, we obtain obviously a non planar graph. Let $h$ be any edge of the graph $H_{1} \backslash E(l)$. There is a path $p_{1} \subset H_{1} \backslash\{E(l) \cup h\}$ such that $H_{2} \cup p_{1}$ contains a graph homeomorphic to $K_{3,3}$. Similarly, for any edge $g$ of the graph $H_{2} \backslash E(l)$ there is a path $p_{2} \subset H_{2} \backslash\{E(l) \cup g\}$ such that $H_{1} \cup p_{2}$ contains a graph homeomorphic to $K_{3,3}$. Therefore removing any edge $k$ from $U \backslash E(l)$ results in a non planar subgraph. On the other hand, it is not difficult to check that removing any edge $e_{i} \in E(l)$ from $U$ also leads to a graph which contains a subgraph homeomorphic to $K_{3,3}$. As an example consider the subgraph $U \backslash e_{5}$ shown in Figure 2.12. The subgraph homeomorphic to $K_{3,3}$ is depicted here by bold line. Therefore $e d(H) \geq 3$. Since $\operatorname{cr}(H)=3$ it follows that $e d(H)=3$.

It is known that the Heawood graph $H$ is toroidal, [19]. The Heawood graph is also cyclically 4 -edge connected. To show this we first note that $H$ is symmetric. Remove an edge $e$ from $H$ as in the proof of Lemma 2.2. 


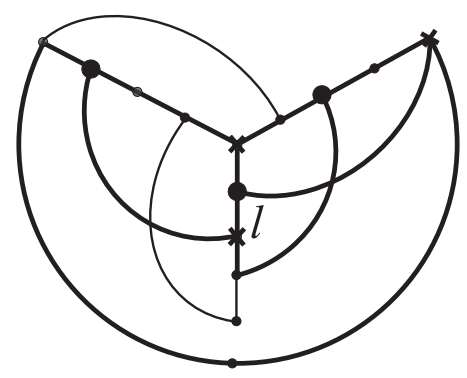

FiguRE 2.12.

Ignoring two vertices of degree 2 in the resulting graph $U^{\prime}$ we shall obtain a cubic graph $U$ of degree 12 , which is homeomorphic to $U^{\prime}$. Evidently, $U$ is 3-connected. It follows that $U$ is cyclically 3 -edge connected, so it $H$ is cyclically 4-edge connected. The last fact will be used in Section 3.

Note also that $\gamma(C N G 3 A)=2$. The proof of this fact will be given in Section 3. It follows that $C N G 3 A$ is a minimal 2-genus graph. It is not difficult to check that $\zeta(C N G 3 A)=3$. By direct computation, the remaining seven 3 -crossing graphs have genus equal to one. We omit here the details of this computation.

4. For $l=4$ there are two minimal crossing graphs: 8-crossed prism graph $\mathrm{Pr}_{8}$, see Figure 2.13a), and the Möbius-Kantor graph $M K$, see Figure 2.13b). By direct computation we have $e d(M K)=3$ and $\operatorname{ed}\left(\operatorname{Pr}_{8}\right)=2$. Moreover it is known that the Möbius-Kantor graph $M K$ is toroidal, [12]. It is not difficult to show that the graph $\mathrm{Pr}_{8}$ is also toroidal.

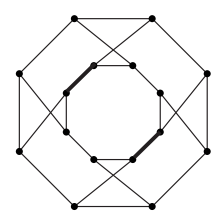

a)

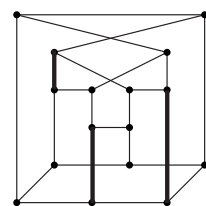

b)

Figure 2.13. Graphs $\mathrm{Pr}_{8}$ and $M K$

\section{AdDitivity OF PARAMETERS $\gamma$ AND ed AND MINIMAL CUBIC GRAPHS}

In this section we introduce two operations on graphs and establish some additivity properties of parameters $e d$ and $\gamma$ with respect to them, in the case of cubic graphs. The first operation is the connected sum of graphs and the second one is the double (crossed) connected sum of them. We also provide some upper bounds for the order of minimal edge deletion and 
minimal genus graphs within the classes of 2-connected and 3-connected cubic graphs.

Let $G_{1}$ and $G_{2}$ be 2-connected cubic graphs with distinguished edges $e$ in $G_{1}$ and $f$ in $G_{2}$. Let $u_{1}, u_{2}$ be the vertices of $e$ and $v_{1}, v_{2}$ the vertices of $f$, respectively. Remove from $G_{1}$ the edge $e$, and from $G_{2}$ the edge $f$. Take the disjoint sum $G$ of resulting graphs, $G=\left(G_{1}-e\right) \sqcup\left(G_{2}-f\right)$, and joint in $G$ the pairs of vertices: $u_{1}$ with $v_{1}$, and $u_{2}$ with $v_{2}$, respectively. Denote the resulting graph by $G_{1} \star G_{2}$. We shall say that $G_{1} \star G_{2}$ is the connected sum of the graphs $G_{1}$ and $G_{2}$ with respect to the pair of edges $e$ and $f$. Note $G_{1} \star G_{2}$ is also 2-connected cubic graph.

Let $G_{1}$ and $G_{2}$ be any two 3 -connected graphs. Take in $G_{1}$ a pair of nonincident edges $\left(e_{1}, e_{2}\right)$, and in $G_{2}$ a pair of nonincident edges $\left(f_{1}, f_{2}\right)$. Denote the vertices of $e_{1}$ by $u_{1}, u_{2}$, and the vertices of $e_{2}$ by $v_{1}, v_{2}$, respectively. Similarly, let $s_{1}, s_{2}$ be the vertices of $f_{1}$, and $t_{1}, t_{2}$ the vertices of $f_{2}$. Delete in $G_{1}$ the edges $e_{1}$ and $e_{2}$, and in $G_{2}$ the edges $f_{1}$ and $f_{2}$. Then take a disjoint $\operatorname{sum} G=\left(G_{1}-e_{1}-e_{2}\right) \sqcup\left(G_{2}-f_{1}-f_{2}\right)$ of two graphs and joint in $G$ the following pairs of vertices: $u_{1}$ and $s_{1}, u_{2}$ and $s_{2}, v_{1}$ and $t_{1}$, and $v_{2}$ and $t_{2}$, respectively. Denote the resulting 2-connected graph $G_{1} * G_{2}$ and call it a double connected sum of $G_{1}$ and $G_{2}$. The four edges joining the graphs $G_{1}-e_{1}-e_{2}$ and $G_{2}-f_{1}-f_{2}$ are called the bridge edges of the graph $G_{1} * G_{2}$ and are denoted $h_{1}, h_{2}, h_{3}$ and $h_{4}$, see Figure 3.1.

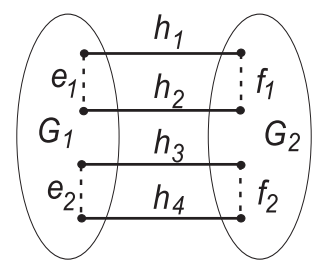

Figure 3.1. A double connected sum of graphs $G_{1}$ and $G_{2}$

If in the above definition we join $u_{1}, u_{2}$ with the vertices incident to different edges $f_{1}$ and $f_{2}$ (then $v_{1}$ and $v_{2}$ are also joined with the vertices of different edges $f_{1}$ and $f_{2}$ ), the resulting cubic graph is called the crossed connected sum of $G_{1}$ and $G_{2}$ and is denoted by $G_{1} \sharp G_{2}$, see Figure 3.2.

It is clear that the operations of double connected sum and crossed connected sums are not determined uniquely and the result $G_{1} * G_{2}$ depends on the distinguished edges of two graphs.

Let $e$ be an edge of the connected cubic graph $G$. We shall say that $e$ is inessential (subject to the parameter $\gamma$ ) if $\gamma(G)=\gamma(G-e)$. Otherwise $e$ is called essential. It is naturally to ask whether the (oriented) genus is additive under taking the operations of connected sum and double connected 


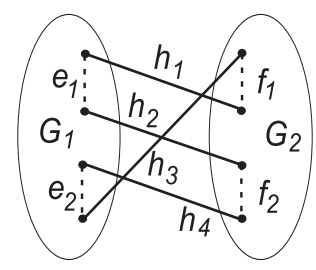

Figure 3.2. The crossed connected sum of graphs $G_{1}$ and $G_{2}$

sum of cubic graphs. In general, the answer is negative. For example, we have $\gamma\left(K_{3,3}\right)=1$ while $\gamma\left(K_{3,3} \star K_{3,3}\right)=1 \neq 2$.

Similarly, the genus is not additive subject to the operation of double connected sum of cubic graphs.

The following assertions show that under certain conditions, the (oriented) genus is subadditive or additive with respect to the operations defined above.

Theorem 3.1. Let $G_{1}$ and $G_{2}$ be 2-connected cubic graphs of genus $k$ and $l$, respectively. Let e and $f$ be distinguished edges of $G_{1}$ and $G_{2}$, respectively and $G_{1} \star G_{2}$ be the connected sum of $G_{1}$ and $G_{2}$. Then

$$
\gamma\left(G_{1}\right)+\gamma\left(G_{2}\right) \geq \gamma\left(G_{1} \star G_{2}\right) \geq \gamma\left(G_{1}\right)+\gamma\left(G_{2}\right)-1 .
$$

Moreover if $e$ is inessential in $G_{1}$ or $f$ is inessential in $G_{2}$, then

$$
\gamma\left(G_{1} \star G_{2}\right)=k+l .
$$

Proof. Let $\varphi_{1}: G_{1} \rightarrow M_{1}$ be a minimal embedding of the graph $G_{1}$ in the surface $M_{1}$ with $\gamma\left(G_{1}\right)=\gamma\left(M_{1}\right)$ and $\varphi_{2}: G_{2} \rightarrow M_{2}$ be a minimal embedding of the graph $G_{2}$ in the surface $M_{2}$ with $\gamma\left(G_{2}\right)=\gamma\left(M_{2}\right)$. Cut an open disc $D_{1}$ in $M_{1}$ containing the edge $e$ of $G_{1}$ and an open disc $D_{2}$ in $M_{2}$ containing the edge $f$ of $G_{2}$. Then join the resulting surfaces $M_{1}^{\prime}$ and $M_{2}^{\prime}$ with a tube $t$, where one connected component of $\partial t$ is identified with $\partial D_{1}$ and the other connected component of $\partial t$ is identified with $\partial D_{2}$. The resulting surface is denoted by $M$. Drawing the bridge edges $h_{1}$ and $h_{2}$ in the tube $t$, we obtain an embedding of the graph $G_{1} \star G_{2}$ into the connected surface $M$. The inequality $\gamma\left(G_{1}\right)+\gamma\left(G_{2}\right) \geq \gamma\left(G_{1} \star G_{2}\right)$ now follows.

We continue with proving the second assertion. Denote the two bridge edges of $G_{1} \star G_{2}$ by $h_{1}$ and $h_{2}$. Let $\varphi: G_{1} \star G_{2} \rightarrow M$ be a minimal embedding of the graph $G_{1} \star G_{2}$ in a closed orientable surface $M$. Then $\varphi$ is a 2cell embedding. Consider a regular neighborhood $N_{1}$ of the polyhedron $\varphi\left(G_{1}\right)-e$ in $M$. Then $N_{1}$ is a compact 2-manifold with the boundary $\partial M$. The compact 2-manifold $M_{1}=\overline{M \backslash N_{1}}$ is decomposed into several connected components $S_{1}, \ldots, S_{l}$. We also have $\partial\left(N_{1}\right)=\partial\left(M_{1}\right)$. The 
connected graph $\varphi\left(G_{2}-f\right)$ is contained in one such connected component, say $S_{1}$. All other connected components of $M \backslash N_{1}$ must be open 2-disks. We have $\partial\left(S_{1}\right)=m_{1} \sqcup m_{2} \sqcup \ldots \sqcup m_{k}$ where each $m_{i}$ is a circle. The 2-manifolds $N_{1}$ and $S_{1}$ have each boundary component $m_{i}$ in common.

We claim that each $m_{i}$ intersects at least one bridge edge $h_{i}$ of $G_{1} \star G_{2}$. Otherwise we can cut the manifold $M$ along the circle $m_{j}$, not intersecting the bridge edges, past the two holes by discs to obtain another closed orientable 2-manifold $M^{\prime}$. Note that $\gamma\left(M^{\prime}\right)=\gamma(M)-1$ and the graph $G_{1} \star G_{2}$ has an embedding in $M^{\prime}$. But this contradicts to the assumption that the embedding $\varphi$ of the graph $G_{1} \star G_{2}$ is minimal.

Denote by $\Pi$ the rotation system on $G_{1} \star G_{2}$ induced by $\varphi$. Consider the facial circuits of the rotation system $\Pi$ that contains bridge edges $h_{1}$ and $h_{2}$. We have the following (alternative) possibilities.

1) There are facial circuits $c_{1}$ and $c_{2}$ of $\Pi$ such that $c_{1}$ contains $h_{1}$ (twice) and $c_{2}$ contains $h_{2}$ (twice). The corresponding closed faces $r_{1}$ and $r_{2}$ bounded by $c_{1}$ and $c_{2}$, respectively, form two handles in $M, H_{1}$ and $H_{2}$. Then $\chi(M) \leq 0$. Cutting $M$ along the meridians $m_{1}$ and $m_{2}$ of $H_{1}$ and $H_{2}$ and pasting the holes by discs, we shall obtain two disjoint closed orientable surfaces, $M_{1}$ and $M_{2}$. This induces embeddings of the graph $G_{1}-e$ in the surface $M_{1}$ and the graph $G_{2}-f$ in the surface $M_{2}$. We have $\gamma(M)=\gamma\left(M_{1}\right)+\gamma\left(M_{2}\right)+1$. Since $\gamma\left(G_{1}-e\right)=\gamma\left(G_{1}\right)$ or $\gamma\left(G_{2}-f\right)=\gamma\left(G_{2}\right)$, the assertion follows.

2) There are two facial circuits $c_{1}$ and $c_{2}$ of $\Pi$ each of which contains both the edges $h_{1}$ and $h_{2}$. Fix an orientation on $M$. Let $r_{1}$ and $r_{2}$ be the faces of the embedding $\varphi$ bounded by $c_{1}$ and $c_{2}$, respectively. The closed faces $r_{1}$ and $r_{2}$ glued along the edges $h_{1}$ and $h_{2}$ form a handle. Removing from $M$ the (open) faces $r_{1}, r_{2}$ together with the edges $h_{1}$ and $h_{2}$, we shall obtain two disjoint 2-manifolds, $M_{1}^{\prime}$ and $M_{2}^{\prime}$ with boundaries $\partial M_{1}^{\prime}$ and $\partial M_{2}^{\prime}$, respectively. Elimination of the edges $h_{1}$ and $h_{2}$ in $G_{1} \star G_{2}$ leads to a surgery of the rotation system $\Pi$ and induces actually the rotation systems $\Pi_{1}$ and $\Pi_{2}$ on the graphs $G_{1}-e$ and $G_{2}-f$, respectively. More precisely, instead of the facial circuits $c_{1}$ and $c_{2}$ in $\Pi$ we have two new circuits, $d_{1}$ and $d_{2}$, respectively, in $\Pi_{1}$ and $\Pi_{2}$. We thus have $\gamma\left(G_{1} \star G_{2}\right) \geq \gamma\left(G_{1}-e\right)+\gamma\left(G_{2}-f\right)$. The rotation systems $\Pi_{1}$ and $\Pi_{2}$ generates embeddings of the graphs $G_{1}-e$ and $G_{2}-f$ in the surfaces $M_{1}$ and $M_{2}$, respectively. By drawing the edge $e$ in the face $D_{1}$ bounded by the circuit $d_{1}$ and the edge $f$ in the disc $D_{2}$ bounded by the circuit $d_{2}$, we obtain embeddings of $G_{1}$ into $M_{1}$ and $G_{2}$ into $M_{2}$. Therefore we have $\gamma(M) \geq \gamma\left(G_{1}\right)+\gamma\left(G_{2}\right)$.

3) There is a unique facial circuit $c$ of $\Pi$ which contains both the edges $h_{1}$ and $h_{2}$ twice. Now we proceed just as in the case 1). After surgery of the surface $M$ we shall obtain two disjoint surfaces, $M_{1}$ and $M_{2}$, such that 
$\gamma(M)=\gamma\left(M_{1}\right)+\gamma\left(M_{2}\right)+1$. Moreover, $G_{1}$ has embedding in $M_{1}$ and $G_{2}$ has embedding in $M_{2}$. Since $\gamma\left(G_{1}-e\right)=\gamma\left(G_{1}\right)$ or $\gamma\left(G_{2}-f\right)=\gamma\left(G_{2}\right)$ we have $\gamma(M) \geq \gamma\left(G_{1}\right)+\gamma\left(G_{2}\right)$ completing the proof of the second assertion.

The inequality $\gamma\left(G_{1} \star G_{2}\right) \geq \gamma\left(G_{1}\right)+\gamma\left(G_{2}\right)-1$ follows directly from the proof of the second assertion through the careful analysis of the cases 1)-3).

Corollary 3.2. Let $G_{1}$ be a 2 -connected cubic graphs with the distinguished edge e. Let $e^{\prime}$ be a distinguished edge of the graph $K_{3,3}$ and $H=G_{1} \star K_{3,3}$ be a connected sum of $G_{1}$ and $K_{3,3}$ subject to the edges $e$ and $e^{\prime}$. If $e$ is inessential in $G_{1}$, then $\gamma(H)=\gamma\left(G_{1}\right)+1$.

Now take in the graph $K_{3,3}$ an edge $e$ and replace it with two parallel edges, $e_{1}$ and $e_{2}$. The resulting cubic graph is denoted by $K_{1}$. It is clear that both $e_{1}$ and $e_{2}$ are inessential in $K_{1}$. Take $e_{1}$ as a distinguished edge of $K_{1}$ and consider the connected sum $K_{2}=K_{1} \star K_{3,3}$. By Corollary 3.2, $\gamma\left(K_{2}\right)=2$. Iterating this process, we obtain a sequence $K_{l}$ of 2 -connected cubic graphs with $\gamma\left(K_{l}\right)=l$. Note that the order of $K_{l}$ is equal to $8 l-2$.

Corollary 3.3. If $H$ is a minimal l-genus graph in the class of 2-connected graphs, then $|H| \leq 8 l-2$.

Theorem 3.4. Let $G_{1}$ be a 3-connected cubic graph with the pair of distinguished edges $e_{1}$ and $e_{2}$ and $G_{2}$ be a cyclically 4-edge connected cubic graph with the pair of distinguished edges $f_{1}$ and $f_{2}$. Assume that $\gamma\left(G_{1}-e_{1}\right)=\gamma\left(G_{1}\right)$ or $\gamma\left(G_{1}-e_{2}\right)=\gamma\left(G_{1}\right)$ and $\gamma\left(G_{2}-\left\{f_{1}, f_{2}\right\}\right) \geq \gamma\left(G_{2}\right)-1$. Then $G_{1} * G_{2}$ is a 3 -connected graph and $\gamma\left(G_{1} * G_{2}\right) \geq \gamma\left(G_{1}\right)+\gamma\left(G_{2}\right)-1$.

Proof. The fact that the graph $G_{1} * G_{2}$ is 3 -connected does not depend on topological properties of graphs $G_{1}$ and $G_{2}$ and actually follows from the proof of Theorem 3.14 (see below).

Let $\psi$ be an embedding of the graph $G_{1} * G_{2}$ in a surface $M$ of minimal genus. Consider a subpolyhedron $P=\psi\left(G_{1}-\left\{e_{1}, e_{2}\right\}\right)$ in $M$. Let $N(P)$ be a regular neighborhood of $P$ in $M$. This is a compact submanifold of $M$ (see, for example [11]) and its boundary $\partial(N(P))$ of $N(P)$ consists of $k$ disjoint circles $c_{i}, \ldots, c_{k}$.

Let $S$ be a complementary submanifold of $N(P)$ in $M$. It consists of several connected components $S_{i}, S=\sqcup_{i} S_{i}$. Since the graph $G_{2}-\left\{f_{1}, f_{2}\right\}$ is connected, it is contained in one such component, say $S_{j}$. Denote by $M_{2}$ the closure of submanifold $S_{j}$ in $M$. We have obviously $\partial M_{2} \subset \partial(N(P))$, so $\partial M_{2}$ is the disjoint union of several circles $c_{i}$, i.e. $\partial M_{2}=c_{i_{1}} \sqcup \ldots \sqcup c_{i_{l}}$, where $l \leq k$. 
As the embedding of $G_{1} * G_{2}$ is genus minimal, all other connected components of $M \backslash N(P)$ must be open 2-disks. Moreover each boundary component $c_{i}$ of $M_{2}$ must intersect at least one bridge edge $h_{1}, h_{2}, h_{3}, h_{4}$. We may suggest without loss of generality that each bridge edge $h_{m}, m=1, \ldots, 4$, of $G_{1} * G_{2}$ intersects in $M$ a unique circle $c_{m}$ at one point and each such intersection is transversal (see also the proof of Theorem 3.5). In particular, $\partial M_{2}$ consists of at most 4 circles i.e. $l \leq 4$.

Denote by $M_{1}$ the submanifold $M \backslash S_{j}$. Both the submanifolds $M_{1}$ and $M_{2}$ are connected and by construction we have $\partial M_{1}=\partial M_{2}=\sqcup_{s=1}^{l} c_{i_{s}}$. Moreover the graph $G_{1}-\left\{e_{1}, e_{2}\right\}$ is embedded in $M_{1}$ and $G_{2}-\left\{f_{1}, f_{2}\right\}$ is embedded in $M_{2}$. Glue the boundary components of $\partial M_{1}$ and $\partial M_{2}$ by discs and denote the obtained surfaces by $M_{1}^{\prime}$ and $M_{2}^{\prime}$, respectively.

Since $G_{1}-\left\{e_{1}, e_{2}\right\} \subseteq M_{1}^{\prime}$, we have that $\gamma\left(M_{1}^{\prime}\right) \geq \gamma\left(G_{1}-\left\{e_{1}, e_{2}\right\}\right)$. Moreover, from the inequality $\gamma\left(G_{1}-e_{1}\right) \leq \gamma\left(G_{1}-\left\{e_{1}, e_{2}\right\}\right)+1$ and assumption $\gamma\left(G_{1}-e_{1}\right)=\gamma\left(G_{1}\right)$ we also get that

$$
\gamma\left(M_{1}^{\prime}\right) \geq \gamma\left(G_{1}-\left\{e_{1}, e_{2}\right\}\right) \geq \gamma\left(G_{1}-e_{1}\right)-1=\gamma\left(G_{1}\right)-1 .
$$

As $G_{2}-\left\{f_{1}, f_{2}\right\} \subseteq M_{2}^{\prime}$, by the assumption $\gamma\left(G_{2}-\left\{f_{1}, f_{2}\right\}\right) \geq \gamma\left(G_{2}\right)-1$, we have

$$
\gamma\left(M_{2}^{\prime}\right) \geq \gamma\left(G_{2}-\left\{f_{1}, f_{2}\right\}\right) \geq \gamma\left(G_{2}\right)-1 .
$$

We have to show that $\gamma(M) \geq \gamma\left(G_{1}\right)+\gamma\left(G_{2}\right)-1$. Suppose

$$
\gamma(M) \geq \gamma\left(G_{1}\right)+\gamma\left(G_{2}\right)-2 \text {. }
$$

By the above reasoning, this is possible whenever we have the following $\gamma\left(M_{1}^{\prime}\right)=\gamma\left(G_{1}\right)-1$ and $\gamma\left(M_{2}^{\prime}\right)=\gamma\left(G_{2}\right)-1$. In other words,

$$
\gamma(M)=\gamma\left(M_{1}\right)+\gamma\left(M_{2}\right)
$$

so $M$ is obtained from $M_{1}$ and $M_{2}$ by gluing along one boundary component. Therefore $\partial M_{1}=\partial M_{2}$ is a circle $c$. In particular, $M_{1}^{\prime}$ is obtained from $M_{1}$ by attaching a disc $D$, so $\gamma\left(M_{1}^{\prime}\right)=\gamma\left(M_{1}\right)$. It follows that the both ends of $e_{2}$ belong to $c$, so one can extend embedding $G_{1}-\left\{e_{1}, e_{2}\right\} \subset M_{1}$ to embedding $G_{1}-e_{1} \subset M_{1}^{\prime}=M_{1} \cup D$ by drawing $e_{2}$ in the closed 2-cell $\bar{D}$. We thus get embedding of $G_{1}-e_{1}$ into surface $M_{1}^{\prime}$ of genus $\gamma\left(G_{1}\right)-1$ contradicting to our assumption.

Note also that an analogue of Theorem 3.4 holds also for crossed connected sum of cubic graphs.

Let $G_{1}$ be a 2-connected cubic graph of genus $k>0$ which has the pair of distinguished non incident edges $\left\{e_{1}=\left(u_{1}, v_{1}\right), e_{2}=\left(u_{2}, v_{2}\right)\right\}$ and let $G_{2}$ be a connected cubic graph with the pair of distinguished non incident edges $\left\{f_{1}=\left(u_{1}^{\prime}, v_{1}^{\prime}\right), f_{2}=\left(u_{2}^{\prime}, v_{2}^{\prime}\right)\right\}$. Assume that the following two conditions holds: 
(i) at least one of the edges $e_{1}, e_{2}$ in $G_{1}$ is inessential;

(ii) $\gamma\left(G_{2}\right)=1$ and either $\gamma\left(G_{2}-\left\{f_{1}, f_{2}\right\}\right)=1$, or $\gamma\left(G_{2}-\left\{f_{1}, f_{2}\right\}\right)=0$ and for any plain embedding of $G_{2}-\left\{f_{1}, f_{2}\right\}$ there is no facial circuit $c^{\prime}$ containing the four vertices $u_{1}^{\prime}, v_{1}^{\prime}, u_{2}^{\prime}, v_{2}^{\prime}$ and the only possibility that the two facial circuits $c_{1}^{\prime}, c_{2}^{\prime}$ cover all these vertices is that one of them contains the vertices $u_{1}^{\prime}, u_{2}^{\prime}$ and the other one contains the vertices $v_{1}^{\prime}, v_{2}^{\prime}$.

For a moment, let $G_{1} \sharp G_{2}$ denote the crossed connected sum of cubic graphs $G_{1}$ and $G_{2}$ in which the vertices of the pair $\left\{u_{1}, v_{1}\right\}$ are joined to the vertices of the pair $\left\{u_{1}^{\prime}, u_{2}^{\prime}\right\}$ and the vertices of the pair $\left\{u_{2}, v_{2}\right\}$ to the vertices of the pair $\left\{v_{1}^{\prime}, v_{2}^{\prime}\right\}$.

Theorem 3.5. Let $G_{1}$ and $G_{2}$ be cubic graphs that satisfy conditions (i) and (ii). Assume that $G_{1}$ is 3-connected and $G_{2}$ is cyclically 4-edge connected. Then $G_{1} \sharp G_{2}$ is 3-connected graph and $\gamma\left(G_{1} \sharp G_{2}\right)=k+1$.

Proof. The proof of the first assertion follows from the proof of the first part of Theorem 3.14.

It remains to prove the second assertion. Suppose that

$$
\gamma\left(G_{1} \sharp G_{2}\right) \leq k .
$$

Let $\varphi$ be an embedding of $G_{1} \sharp G_{2}$ into an orientable surface $M$ of genus $k$, and $\psi$ be the embedding of the subgraph $G_{1}-\left\{e_{1}, e_{2}\right\}$ into $M$ induced by the embedding $\varphi$. Let also $N\left(G_{1}\right)$ be an open regular neighborhood of the polyhedron $\psi\left(G_{1}-\left\{e_{1}, e_{2}\right\}\right)$ in $M$.

Let $s$ be a connected component of the 2-manifold $M_{2}=M \backslash N\left(G_{1}\right)$ containing the image $\varphi\left(G_{2}-\left\{f_{1}, f_{2}\right\}\right)$. Then $s$ cannot be a disc (i.e. a face of the embedding $\psi$ ). Indeed, otherwise the bridge edges of $G_{1} \sharp G_{2}$ would join the four vertices from $G_{2}-\left\{f_{1}, f_{2}\right\}$ to four vertices of $G_{1}-\left\{e_{1}, e_{2}\right\}$ in a disc. But this is impossible by condition (ii). Therefore $s$ contains tubes (i.e. is a submanifold with nontrivial fundamental group). It follows that $\gamma\left(G_{1} \sharp G_{2}\right) \geq k$.

It can occur that $\partial s$ consists of one connected component, a circle $c$. Then $M_{1}=\overline{M \backslash s}$ is a 2-manifold with the boundary $\partial M_{1}=c$. After gluing a disc $D$ to $M_{1}$ along the circle $c$ we shall obtain a surface $T$ of genus $k-1$. In this case we can draw the edge $e_{1}$ (or the edge $e_{2}$ ) in the disc $D$ and obtain an embedding of the graph $G_{1}-e_{1}$ into the surface $M_{1}$ contradicting with the equality $\gamma\left(G_{1}-e_{1}\right)=k$. We thus exclude this possibility.

Suppose now that $s$ is glued to the rest of the surface $M$ along two or more circles $c_{i}$. The number of circles cannot be bigger than two, otherwise the genus of $M$ would be greater than $k$, contradicting to our assumption. 
Assume that $s$ has two boundary components, $c_{1}$ and $c_{2}$. Then $s$ is a cylinder and $\gamma(\overline{M \backslash s})=k-1$. There are two tubes $t_{1}$ and $t_{2}$ inside $s$ which contain four bridge edges of the graph $G_{1} \sharp G_{2}$. A tube $t_{i}, i=1,2$, cannot contain three bridge edges $h_{l}$, otherwise one circle $c_{i}^{\prime}$ would contain three vertices from the set $L=\left\{u_{1}^{\prime}, v_{1}^{\prime}, u_{2}^{\prime}, v_{2}^{\prime}\right\}$ and the other circle $c_{3-i}^{\prime}$ contains the remaining vertex, which is impossible by condition (ii).

Therefore the first tube $t_{1}$, bounded by $c_{1}$ on one side, contains two bridge edges $h_{1}$ and $h_{2}$ joining the ends of the edge $e_{1}$ to the vertices, say $u_{1}^{\prime}$ and $u_{2}^{\prime}$, positioned on the facial circuit $c_{1}^{\prime}$ of $G_{2}-f_{1}-f_{2}$.

Similarly, the second tube $t_{2}$, bounded by $c_{2}$ on one side, contains the remaining bridge edges $h_{3}$ and $h_{4}$ which join the ends of the edge $e_{2}$ to the vertices $v_{1}^{\prime}$ and $v_{2}^{\prime}$, positioned on the second facial circuit $c_{2}^{\prime}$ of $G_{2}-f_{1}-f_{2}$, see Figure 3.3.

In this case we can add the edges $e_{1}=\left(u_{1}, v_{1}\right)$ and $e_{2}=\left(u_{2}, v_{2}\right)$ to the subgraph $G_{1}-e_{1}-e_{2}$ and draw them in the 2-manifold $N\left(G_{1}\right)$. It follows that the graph $G_{1}$ admits embedding in a surface of genus $k-1$ contradicting to the condition (i). This completes the proof of the second assertion.

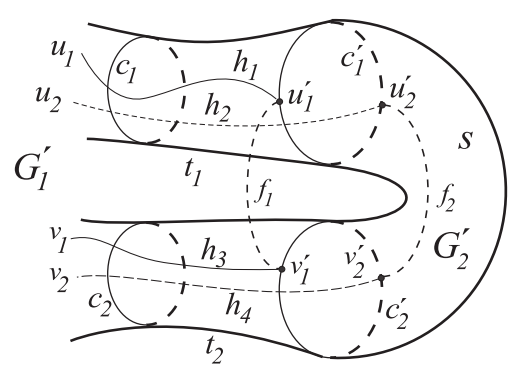

FiguRE 3.3.

Example 3.6. Consider the cubic graph $K$ obtained from $K_{3,3}$ by doubling an edge $e$. Instead of $e$, we have in $K$ two edges $e_{1}$ and $e_{2}$, see Figure 3.4. Take the edges $e_{1}$ and $e_{2}$ to be distinguished in $K$. Removing $e_{1}$ and $e_{2}$ from $K$ we shall obtain a subcubic graph $K^{\prime}$. Obviously, $K^{\prime}$ is homeomorphic to the complete graph $K_{4}$ so there is a unique embedding $\rho$ of $K^{\prime}$ in the sphere $S^{2}$. The pairs of vertices $\left\{u_{1}, u_{2}\right\}$ and $\left\{v_{1}, v_{2}\right\}$ are positioned on two different faces of $\rho$ and there is no face $r$ of $\rho$ that contains three of these vertices in the boundary. It follows that $K$ satisfies condition (i) (subject to the pair of edges $e_{1}$ and $\left.e_{2}\right)$. It is also clear that $K$ satisfies the condition (i) as well (subject to the pair of edges $e_{1}$ and $e_{2}$ ). 
It follows that $\gamma(K \sharp K)=2$. Note also that $K \sharp K$ is cyclically 4-edge connected cubic graph.

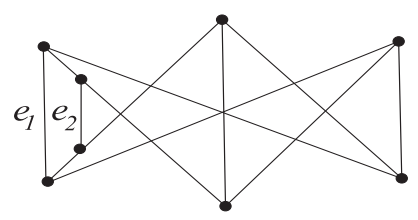

Figure 3.4. The cubic graph $K$

Lemma 3.7. The genus of cubic graph $C N G 3 A$ is equal to 2 .

Proof. Cut the graph $C N G 3 A$ across four edges as shown in Figure 3.5. We have a decomposition of $C N G 3 A$ into two planar graphs $G_{1}$ and $G_{2}$ such that $G_{1}$ contains four semiedges $e_{1}, e_{2}, e_{3}$ and $e_{4}$ and $G_{2}$ contains four semiedges $f_{1}, f_{2}, f_{3}$ and $f_{4}$.
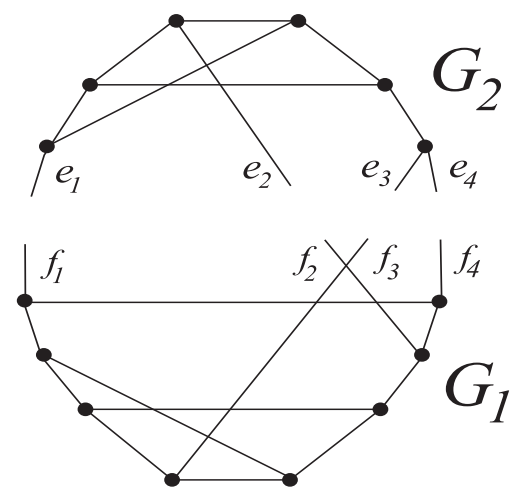

FIGURE 3.5.

Suppose that the graph $C N G 3 A$ is toroidal. Let $\varphi$ denote embedding of this graph in the torus $T$. Then $\varphi$ induces embeddings $\varphi_{1}$ and $\varphi_{2}$ of the subgraphs $G_{1}$ and $G_{2}$, respectively, in the torus. Let $N_{1}$ and $N_{2}$ be open regular neighborhoods of the graphs $\varphi_{1}\left(G_{1}\right)$ and $\varphi_{1}\left(G_{2}\right)$, respectively, in $T$. Then $G_{1}$ is contained in one connected component $t$ of the 2-manifold $T \backslash N_{2}$ and $G_{2}$ is contained in one connected component $s$ of the 2-manifold $\overline{T \backslash N_{1}}$. The component $t$ cannot be a disc since there is no planar embedding of $G_{1}$ which contains all semiedges inside the same region $r$. Similarly the component $s$ is not a disc. Therefore the only possibility to obtain embedding of the graph $C N G 3 A$ in the torus is as follows. The subgraph 
$G_{1}$ is embedding into a sphere $S_{1}$ with two holes, the subgraph $G_{1}$ is embedding into a sphere $S_{2}$ with two holes and the spheres $S_{1}$ and $S_{2}$ are joining by two tubes $\tau_{1}$ and $\tau_{2}$ which contain four pairs of glued semiedges: $\left(e_{1}, f_{1}\right),\left(e_{2}, f_{2}\right),\left(e_{3}, f_{3}\right)$ and $\left(e_{4}, f_{4}\right)$. By careful inspection all possibilities we can easily check that this is impossible.

Now starting from the graphs $C N G 3 A$ and $K$ in Example 3.6, we can inductively construct a sequence of 3-connected cubic graphs $H_{l}$ of order 8l. Note that at each inductive step $l$, there is at least two nonincident inessential edges in $H_{l}$. By Lemma 3.7 and Theorem 3.5 we have $\gamma\left(H_{l}\right)=l$.

Corollary 3.8. If $H$ is minimal l-genus graph in the class of 3-connected cubic graphs, then $|H| \leq 8$ l.

Denote by $\chi^{\prime}(G)$ the chromatic index of the graph $G$. A cubic graph $G$ is called colorable if $\chi^{\prime}(G)=3$, otherwise $G$ is called uncolorable (i.e. $\left.\chi^{\prime}(G)=4\right)$ or a weak snark. A weak snark which is cyclically 4-edge connected and whose girth is at least five is called a snark, [15].

The Petersen graph is a simplest example of a snark. Using the operation of dot product, see Figure 3.6, one obtains from any two snarks of orders $k$ and $l$, respectively, a bigger snark of order $k+l-2$. Note that the dot product $G_{1} \cdot G_{2}$ of two cubic graphs $G_{1}$ and $G_{2}$ is defined non uniquely.
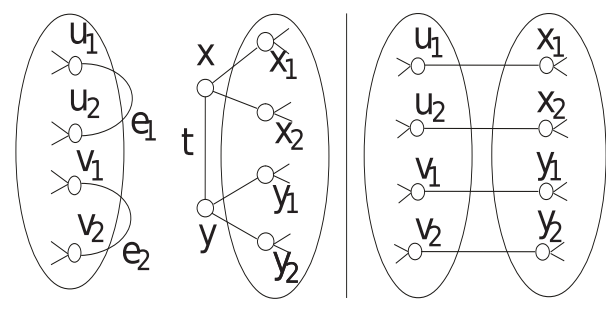

FiguRE 3.6. The dot product of two snarks

In [15] the authors consider different powers $P^{k}$ of the Petersen graph $P$ and study their genus. A $k$-th power $P^{k}$ of the Petersen graph $P$ is defined inductively: $P^{k}=P \cdot P^{k-1}$, where $\cdot$ denote a dot product of the cubic graphs. Since the dot product of two cubic graphs is defined non uniquely, there are several powers $P^{n}$ of the snark $P$ for each natural number $n \geq 2$.

In [15] the authors construct for each pair $(k, n)$ of natural numbers $k$ and $n$, where $k \leq n$ and $k, n \geq 1$, the powers $P^{n}$ such that $\gamma\left(P^{n}\right)=k$. Note that the order of $P^{n}$ is equal to $8 n+2$. This is an open problem to evaluate the number $e d\left(P^{n}\right)$ of the powers $P^{n}$ of $P$ such that $\gamma\left(P^{n}\right)=k$.

In the remaining part of this section, we study additivity properties of the parameter $e d$ subject to operations of connected and double connected sum 
of graphs within the classes of 2-connected and 3-connected cubic graphs. A simple example shows that this parameter is not additive under the connected sum of cubic graphs. It suffice to consider the graphs $K_{3,3} * K_{3,3}$ and $K_{3,3} \star K_{3,3}$. Indeed we have $\operatorname{ed}\left(K_{3,3}\right)=1$ and $\operatorname{ed}\left(K_{3,3} \star K_{3,3}\right)=1$. Moreover $\operatorname{ed}\left(K_{3,3} * K_{3,3}\right)=1$ for appropriate choice of pairs of the non incident edges in the first and second copies of $K_{3,3}$. However under certain conditions an analogue of additivity property holds also for the parameter ed.

Let $G$ be a cubic graph and $e$ and $f$ are two distinguished edges of $G$. We shall say that the edge $e$ of $G$ is inessential (subject to the characteristic $e d)$ if $e d(G-e)=e d(G)$.

Example 3.9. Let $H$ denote the Heawood graph. Take any two edges $e$ and $f$ in $H$, subdivide $e$ with the vertex $x$ and $f$ with the vertex $y$ and connect the new vertices with an edge $h$. Denote the resulting cubic graph by $T$. We claim that the edge $h$ is inessential in $T$. To show this note that $e d(T)=3$. Indeed the equality $e d(T)=4$ would imply that $\operatorname{cr}(T)=4$. But the only 4-crossing minimal cubic graphs are $\operatorname{Pr}_{8}$ and $M K$. As was mentioned in Section 2, the following equalities hold: $e d\left(P r_{8}\right)=2$ and $e d(M K)=3$. Therefore, we have $e d(T)=3$. Notice that $T$ cannot be the graph $M K$, since the latter graph is symmetric, so removing any edge from it and ignoring two new vertices of degree 2 , we obtain a cubic graph $U$ of degree 14 and with the girth equal to five. But it is well known that $H$ is of girth six. Note however that $h$ is a unique inessential edge in $T$, since removal any other edge in $T$ leads to a cubic graph of order 14 that is not isomorphic to $H$, so to a graph $L$ with $\operatorname{ed}(L)=2$.

Example 3.10. Take in the Heawood graph $H$ an edge $e$ and replace it with two parallel edges, $e^{\prime}$ and $e^{\prime \prime}$. Let $H^{\prime}$ denote the resulting cubic graph of order 16. Then both $e^{\prime}$ and $e^{\prime \prime}$ are obviously inessential edges of $H^{\prime}$.

Theorem 3.11. Let $G_{1}$ and $G_{2}$ be two 2-connected cubic graphs with the distinguished edges e in $G_{1}$ and $f$ in $G_{2}$, respectively. If ed $\left(G_{1}\right)=k>0$ and ed $\left(G_{2}\right)=l>0$, then ed $\left(G_{1} \star G_{2}\right) \geq k+l-1$. Moreover if $e$ is inessential in $G_{1}$ and $f$ is inessential in $G_{2}$, then $\operatorname{ed}\left(G_{1} \star G_{2}\right)=k+l$.

Proof. Denote the vertices of $e$ in $G_{1}$ by $u_{1}$ and $u_{2}$ and the vertices of $f$ in $G_{2}$ by $v_{1}$ and $v_{2}$. Put $e d\left(G_{1} \star G_{2}\right)=m$. Let $E=\left\{e_{1}, \ldots, e_{m}\right\}$ be the minimal set of edges in $G_{1} \star G_{2}$ such that $G_{1} \star G_{2}-E$ is planar. By minimality of $E$, the graph $G_{1} \star G_{2}-E$ is connected.

Assume that $E$ contains neither $t_{1}=\left(u_{1}, v_{1}\right)$ nor $t_{2}=\left(u_{2}, v_{2}\right)$. Then either there exists a path $p_{1}$ joining $u_{1}$ to $u_{2}$ in $G_{1}-\left\{e, e_{1}, \ldots, e_{m}\right\}$ or a path $p_{2}$ in $G_{2}-\left\{f, e_{1}, \ldots, e_{m}\right\}$ joining $v_{1}$ to $v_{2}$. 
Suppose that the first possibility occurs. Then $p_{1}$ together with the edges $t_{1}$ and $t_{2}$ form in $G_{1} \star G_{2}$ a path $p$ that joins $v_{1}$ to $v_{2}$ and do not intersect $\left(G_{2}-f\right)$, for exception the end vertices $v_{1}$ and $v_{2}$. Evidently, $p$ is homeomorphic to the removed edge $f$ in $G_{2}$ and replaces actually it. Since $G_{2}-\left\{f, e_{1}, \ldots, e_{m}\right\} \cup p$ is planar, it follows that $\left|E\left(G_{2}-f\right) \cap E\right| \geq l$. As $\left|E\left(G_{1}-e\right) \cap E\right| \geq k-1$ and $\left|E\left(G_{2}-f\right) \cap E\right| \geq l$, the inequality $|E| \geq k+l-1$ follows. In the second case, we have $\left|E\left(G_{1}-e\right) \cap E\right| \geq k$ and the assertion also follows.

Assume now that $E$ contains one of the edges $t_{1}$ or $t_{2}$. Then $E$ contains at least $k-1$ edges of $G_{1}-e$ and $l-1$ edges of $G_{2}-f$, and the first assertion follows.

The second assertion of the theorem follows directly from the definitions of the connected sum of cubic graphs and the minimal edge deletion set.

Let $G$ be a connected cubic graph with $\operatorname{ed}(G)=l$ and $L \subset E(G)$ be an edge deletion subset of $G$, so the graph $G-L$ is planar. Note that if the subgraph $G-L$ is disconnected, then $|L| \geq l+1$. Indeed, suppose contrary that $|L|=l$. We can add some edge $r$ from $L$ to the graph $G-L$ and obtain a planar subgraph $U$ of $G$. But this contradicts to the assumption that $e d(G)=l$.

Let $G$ be a cyclically 4 -edge connected cubic graph with $\operatorname{ed}(G)=l$. Let also $e_{2}=\left(u_{2}, u_{2}^{\prime}\right)$ and $f_{2}=\left(v_{2}, v_{2}^{\prime}\right)$ be a pair of non incident distinguished and inessential edges in $G$. Put $G^{\prime}=G-\left\{e_{2}, f_{2}\right\}$. Consider an l-cut $L$ in $G^{\prime}$ which decomposes the graph $G^{\prime}$ into two planar components, say $G_{1}^{\prime}$ and $G_{2}^{\prime}$. It may occur that in a plane embedding of $G_{1}^{\prime} \sqcup G_{2}^{\prime}$ the pair of vertices $\left\{u_{2}, v_{2}\right\}\left(\left\{u_{2}, v_{2}^{\prime}\right\}\right.$, respectively) are in the same facial cycle of $G_{1}^{\prime}$ and the pair of vertices $\left\{u_{2}^{\prime}, v_{2}^{\prime}\right\}\left(\left\{u_{2}^{\prime}, v_{2}\right\}\right.$, respectively) are in the same facial cycle of $G_{2}^{\prime}$. Then $L$ is called a cut separating $\left\{u_{2}, v_{2}\right\}$ from $\left\{u_{2}^{\prime}, v_{2}^{\prime}\right\}$ $\left(\left\{u_{2}, v_{2}^{\prime}\right\}\right.$ from $\left\{u_{2}^{\prime}, v_{2}\right\}$, respectively). We shall say that $G$ has the property $P$ (subject to the pair of edges $e_{2}$ and $f_{2}$ ) if no such separating $l$-cut $L$ exists in $G^{\prime}$.

Example 3.12. Let $H^{\prime}$ be a cubic graph as in Example 3.10 and

$$
\left\{e^{\prime}=\left(u, u^{\prime}\right), \quad e^{\prime \prime}=\left(v, v^{\prime}\right)\right\}
$$

be a pair of distinguished edges in $H^{\prime}$. We assert that $H^{\prime}$ has the property $P$ subject to the pair of edges $\left\{e^{\prime}, e^{\prime \prime}\right\}$. Indeed, first note that $H^{\prime}$ is cyclically 4-edge connected and $e d\left(H^{\prime}\right)=3$. Remove the edges $e^{\prime}$ and $e^{\prime \prime}$ from $H^{\prime}$ and denote the resulting graph $G^{\prime}$, see Figure 3.7.

The graph $G^{\prime}$ is also cyclically 4-edge connected and $\operatorname{ed}\left(G^{\prime}\right)=2$. Indeed, suppose that $G$ does not have the property $P$. Let $L$ be a 3-cut of $G^{\prime}$ that separates $\{u, v\}$ from $\left\{u^{\prime}, v^{\prime}\right\}$ and $G_{1}$ and $G_{2}$ the corresponding planar components of $G^{\prime}-L$ such that $u, v \in G_{1}$ and $u^{\prime}, v^{\prime} \in G_{2}$. The only 


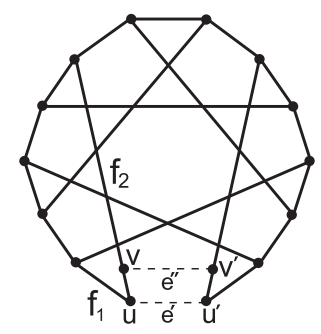

FiguRE 3.7.

possibility for such a cut is that $L$ contains both the edges $f_{1}, f_{2}$ (so $G_{1}$ is simply $K_{2}$ ) and some other edge $f$ of $G^{\prime}$. Consider the component $K$ of $G^{\prime}-\left\{f_{1}, f_{2}\right\}$ that contains the pair of vertices $\left\{u^{\prime}, v^{\prime}\right\} . K$ is of order 14 and contains four vertices of degree two, see Figure 3.8 .

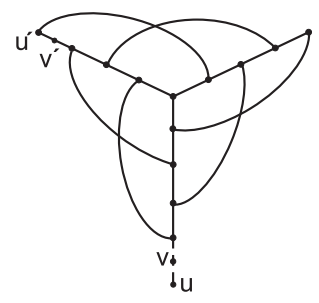

Figure 3.8.

We can ignore the vertices of degree two in $K$ and consider the corresponding cubic graph $K^{\prime}$ of degree 10 which is homeomorphic to $K$. It is easy to see that $g\left(K^{\prime}\right)=5$. It is known that the only cubic graph of degree 10 and of girth 5 is the Petersen graph $P$. However $\operatorname{ed}(P)=2$, so removal any edge $u$ from it does not lead to a planar graph. It follows that removal any edge $f$ from $K$ does not lead to a planar graph. This contradicts to our assumption that $G_{2}=K-f$ is planar. It is rather obvious fact that there is no 3-cut in $G^{\prime}$ separating $\left\{u, v^{\prime}\right\}$ from $\left\{u^{\prime}, v\right\}$. Therefore $H^{\prime}$ has the property $P$ subject to the pair of edges $\left\{e^{\prime}, e^{\prime \prime}\right\}$.

Example 3.13. Let $M K$ denote the Möbius-Kantor graph. It is well known that $M K$ is a symmetric graph. Take in the graph $M K$ any edge $e$ remove it from $M K$. Forgetting two vertices of degree 2 in the resulting graph, we obtain a cubic graph $U$ of order 14 with $g(U)=5$. Then $U$ is not isomorphic to the Heawood graph, so by the results of Section 2 we have $e d(U)=2$. It follows that each edge of $M K$ is essential. ow replace the edge $e$ in $M K$ with two parallel edges $\left\{e^{\prime}=\left(u, u^{\prime}\right), e^{\prime \prime}=\left(v, v^{\prime}\right)\right\}$. Denote the resulting graph by $W$. It is easy to see that both $e^{\prime}$ and $e^{\prime \prime}$ are 
inessential edges of $W$. Let $e^{\prime}$ and $e^{\prime \prime}$ be the distinguished edges of $W$. By the construction, $W$ is cyclically 4 -edge connected and $e d(W)=3$.

Moreover, we assert that $W$ has the property $P$ subject to the pair of edges $\left\{e^{\prime}, e^{\prime \prime}\right\}$. Indeed, suppose the contrary. Remove the edges $e^{\prime}$ and $e^{\prime \prime}$ from $W$ and denote the resulting graph $W^{\prime}$, see Figure 3.9. Since $W^{\prime}$ differs from $U$ by subdivision only, it is cyclically 4 -edge connected and we have $e d\left(W^{\prime}\right)=2$.

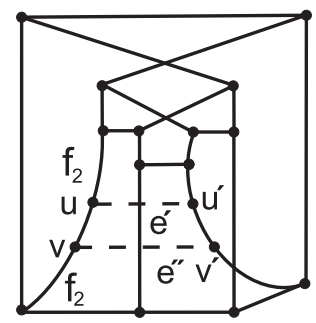

FiguRE 3.9 .

Let $M$ be a 3 -cut of $W^{\prime}$ that separates $\{u, v\}$ from $\left\{u^{\prime}, v^{\prime}\right\}$ and $W_{1}$ and $W_{2}$ the corresponding planar components of $W^{\prime}-M$ where $u, v \in W_{1}$ and $u^{\prime}, v^{\prime} \in W_{2}$. The only possibility for such a cut is that $M$ contains both the edges $f_{1}, f_{2}$ (so $W_{1}$ is simply $K_{2}$ ) and some other edge $f$ of $W^{\prime}$. Consider the component $R$ of $W^{\prime}-\left\{f_{1}, f_{2}\right\}$ that contains the pair of vertices $\left\{u^{\prime}, v^{\prime}\right\}$ (up to homeomorphism of graphs, to obtain $R$ from $M K$ we simply remove from it a star of vertex i.e. the graph $\left.K_{1,3}\right)$. As before, we can forget the vertices $u^{\prime}$ and $v^{\prime}$ of degree two in $R$ and consider the corresponding cubic graph $R^{\prime}$ of degree 12 that is homeomorphic to $R$. It is easy to see that $g\left(R^{\prime}\right)=5$, see Figure 3.10. It is not difficult to check that $\operatorname{ed}\left(R^{\prime}\right)=2$. It follows that removing an edge $f$ from $R$ does not lead to a planar graph. This contradicts to our assumption that $W_{2}=R-f$ is planar. It is rather an obvious fact that there is no 3 -cut in $W^{\prime}$ separating $\left\{u, v^{\prime}\right\}$ from $\left\{u^{\prime}, v\right\}$. Therefore $W$ has the property $P$ subject to the pair of edges $\left\{e^{\prime}, e^{\prime \prime}\right\}$.

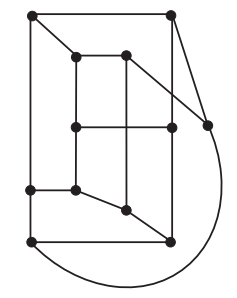

FIGURE 3.10. 
Theorem 3.14. Let $G_{1}$ be a 3-connected cubic graph with ed $\left(G_{1}\right)=k>0$ and $G_{2}$ a cyclically 4-edge connected cubic graph with ed $\left(G_{2}\right)=l>0$. Let $\left\{e_{1}, f_{1}\right\}$ be a pair of distinguished non incident edges in $G_{1}$ and $\left\{e_{2}, f_{2}\right\}$ a pair of non incident distinguished edges in $G_{2}$. Assume that in both the pairs each edge is inessential and $G_{1}$ has the property $P$ with respect to the pair $\left\{e_{1}, f_{1}\right\}$ and $G_{2}$ has the property $P$ with respect to the pair $\left\{e_{2}, f_{2}\right\}$. Then $G_{1} * G_{2}$ is a 3 -connected cubic graph and ed $\left(G_{1} * G_{2}\right) \geq k+l$.

Proof. Let $e_{1}=\left(u_{1}, u_{1}^{\prime}\right), f_{1}=\left(v_{1}, v_{1}^{\prime}\right), e_{2}=\left(u_{2}, u_{2}^{\prime}\right)$ and $f_{2}=\left(v_{2}, v_{2}^{\prime}\right)$. The bridge edges in the graph $H=G_{1} * G_{2}$ are the following:

$$
h_{1}=\left(u_{1}, u_{2}\right), \quad h_{2}=\left(u_{1}^{\prime}, u_{2}^{\prime}\right), \quad h_{3}=\left(v_{1}, v_{2}\right), \quad h_{4}=\left(v_{1}^{\prime}, v_{2}^{\prime}\right) .
$$

The subgraph of $H$ formed by the bridge edges $h_{1}, h_{2}, h_{3}, h_{4}$ is denoted by $B$. Put $G_{1}^{\prime}=G_{1}-\left\{e_{1}, f_{1}\right\}$ and $G_{2}^{\prime}=G_{2}-\left\{e_{2}, f_{2}\right\}$.

Note that if $G$ is cyclically 4 -edge connected cubic graph, then any 3-edge cut of $G$ is of kind $K_{1,3}$ (see, for example, [22]). We claim that the graph $H$ is 3-connected. Suppose contrary that $\lambda(H)=2$. Let $A=\left\{a_{1}, a_{2}\right\}$ be a cut of $H$ consisting of two edges $a_{1}$ and $a_{2}$. Depending on the positions of edges $a_{1}$ and $a_{2}$ in $H$, the following situations can occur.

1) $a_{1} \in E\left(G_{1}^{\prime}\right)$ and $a_{2} \in E\left(G_{2}^{\prime}\right)$. Under this assumption we have

$$
\lambda\left(G_{1}^{\prime}\right)=\lambda\left(G_{2}^{\prime}\right)=1,
$$

so the graph $H$ is decomposed into two components by removing the edges $a_{1}$ and $a_{2}$ as shown in the Figure 3.11. It follows that $E_{1}=\left\{e_{1}, f_{1}, a_{1}\right\}$ is a 3 -cut of the graph $G_{1}$ and $E_{2}=\left\{e_{2}, f_{2}, a_{2}\right\}$ is a 3 -cut of the graph $G_{2}$. However since $G_{2}$ is cyclically 4-edge connected, this is impossible because the graph formed by the set of edges $E_{2}$ is not isomorphic to $K_{1,3}$.

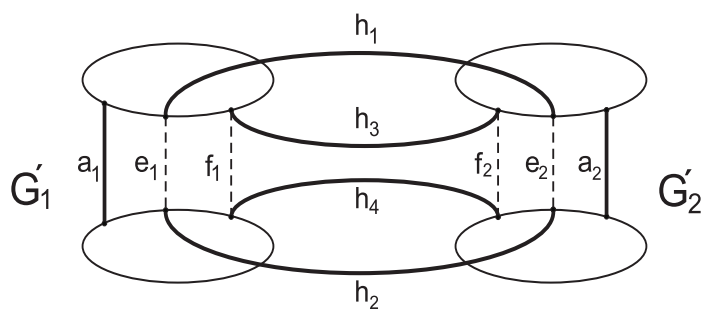

FiguRE 3.11.

2) Both $a_{1}$ and $a_{2}$ are the edges of $G_{1}^{\prime}$. In this case, there are paths $p_{1}$ and $p_{2}$ in $H-E\left(G_{1}^{\prime}\right)$ that join the vertices $u_{1}, u_{1}^{\prime}$ and $v_{1}, v_{1}^{\prime}$, respectively. This means that $U$ decomposes $G_{1}^{\prime}$ into two subgraphs, say $T_{1}$ and $T_{2}$, so that one such $T_{i}$ contains all the vertices $u_{1}, u_{1}^{\prime}, v_{1}, v_{1}^{\prime}$. This would imply that $\lambda\left(G_{1}\right)=2$ contradicting to our assumption. 
3) Both $a_{1}$ and $a_{2}$ are the edges of $G_{2}^{\prime}$. This case can be handled just in the same way as the case 2 ).

Finally, it is obviously that $H$ does not possesses a 2-cut which contains at least one bridge edge. Therefore $H$ is 3 -connected. It remains to show that $e d\left(G_{1} * G_{2}\right) \geq k+l$.

Let $R=\left\{r_{1}, \ldots r_{s}\right\}$ be a minimal edge deletion set in $H$. Denote by $q$ the number $\left|R \cap\left\{h_{1}, h_{2}, h_{3}, h_{4}\right\}\right|$. The planar graph $H-R$ is connected. The proof of the inequality is actually reduced to analyzing the following three cases:

Case 1) $q \geq 2$. By assumption, we have

$$
e d\left(G_{1}^{\prime}\right) \geq k-1 \quad \text { and } \quad e d\left(G_{2}^{\prime}\right) \geq l-1 .
$$

Since the graphs $G_{1}^{\prime}$ and $G_{2}^{\prime}$ are planar, it follows that

$$
\left|E\left(G_{1}^{\prime}\right) \cap R\right| \geq \operatorname{ed}\left(G_{1}^{\prime}\right) \geq k-1, \quad\left|E\left(G_{2}^{\prime}\right) \cap R\right| \geq \operatorname{ed}\left(G_{2}^{\prime}\right) \geq l-1 .
$$

As the sets $E\left(G_{1}^{\prime}\right) \cap R$ and $E\left(G_{1}^{\prime}\right) \cap R$ are disjoint, we have

$$
|R| \geq k-1+l-1+q \geq k+l,
$$

so $e d(H) \geq k+l$.

Case 2) $q=0$, i.e. $R$ does not contain any bridge edge $h_{i}$. Consider a planar drawing $g$ of the connected graph $H-R$. Let $g_{1}$ and $g_{2}$ be the planar embeddings of the subgraphs $G_{1}^{\prime}-R$ and $G_{2}^{\prime}-R$, respectively, induced by $g$. To prove the assertion in this case we have to inspect the following three subcases.

(i) Both the subgraphs $G_{1}^{\prime}-R$ and $G_{2}^{\prime}-R$ are connected. Since $G_{1}^{\prime}-R$ is connected, the plane subgraph $D_{2}=\left(G_{2}^{\prime} \cup B\right)-R$ is contained in a face $\mu$ of the plane embedding $g_{1}$ of the graph $G_{1}^{\prime}-R$. This means that the vertices $u_{1}, u_{1}^{\prime}, v_{1}, v_{1}^{\prime}$ of $G_{1}^{\prime}-R$ are situated on the same facial circuit $c$, the circuit that bounds the face $\mu$. We can draw the edge $e_{1}$ (or the edge $f_{1}$ ) in the face $\mu$ and obtain a planar embedding of the subgraph $G_{1}-\left(f_{1} \cup R\right)$, see Figure 3.12. Since the edge $f_{1}$ is inessential in $G_{1}$, we have $\left|E\left(G_{1}^{\prime}\right) \cap R\right| \geq k$. In the same way we can prove that $\left|E\left(G_{2}^{\prime}\right) \cap R\right| \geq l$. It follows that $|R| \geq k+l$.

(ii) Both the subgraphs $G_{1}^{\prime}-R$ and $G_{2}^{\prime}-R$ are disconnected. Then $\left|E\left(G_{2}^{\prime}\right) \cap R\right| \geq l$ and $\left|E\left(G_{1}^{\prime}\right) \cap R\right| \geq k$, so $|R|=k+l$;

(iii) One of the subgraphs $G_{1}^{\prime}-R$ and $G_{2}^{\prime}-R$ is connected and the other is disconnected. Suppose for instance that $G_{1}^{\prime}-R$ is connected and $G_{2}^{\prime}-R$ is disconnected. Then $G_{2}^{\prime}-R$ consists of two connected components, say $U_{1}$ and $U_{2}$. Since $G_{2}^{\prime}-R$ is disconnected, we have $\left|E\left(G_{2}^{\prime}\right) \cap R\right|=l$. If $\left|E\left(G_{1}^{\prime}\right) \cap R\right|=k$ we have $|R|=k+l$. Suppose that $\left|E\left(G_{1}^{\prime}\right) \cap R\right|=k-1$. Then in any plane embedding of $W=G_{1}^{\prime}-R$, the pair of vertices $u_{1}$ and $u_{1}^{\prime}$ and the pair of vertices $v_{1}, v_{1}^{\prime}$ cannot be neighboring (positioned on the 


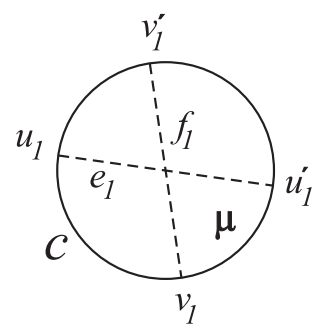

Figure 3.12.

same face). For the given plane subgraphs $G_{2}^{\prime}-R$ and $G_{1}^{\prime}-R$, the only possibility to draw the graph $H$ in the plane is to take a face $c_{1}$ of $G_{1}^{\prime}-R$ containing $u_{1}$ and $v_{1}$, a face $c_{2}$ containing $u_{1}^{\prime}$ and $v_{1}^{\prime}$ (if such exist), insert a connected component $U_{i}$ into the face $c_{1}$, the second connected component $U_{3-i}$ into the face $c_{2}$, then connect the vertices $u_{1}$ with $u_{2}$ and the vertices $v_{1}$ with $v_{2}$ with bridge edges $h_{1}$ and $h_{3}$ inside $c_{1}$, and the vertices $u_{1}^{\prime}$ with $u_{2}^{\prime}$ and the vertices $v_{1}^{\prime}$ with $v_{2}^{\prime}$ inside the face $c_{2}$ with bridge edges $h_{2}$ and $h_{4}$, see Figure 3.13. This is possible only if $u_{2}$ and $v_{2}$ are vertices of the same face of the plane graph $U_{i}$ and $u_{2}^{\prime}$ and $v_{2}^{\prime}$ are vertices of the same face of the plane graph $U_{3-i}$. However the latter is excluded by property $P$. Therefore $\left|E\left(G_{1}^{\prime}\right) \cap R\right|=k$ and $|R|=k+l$.

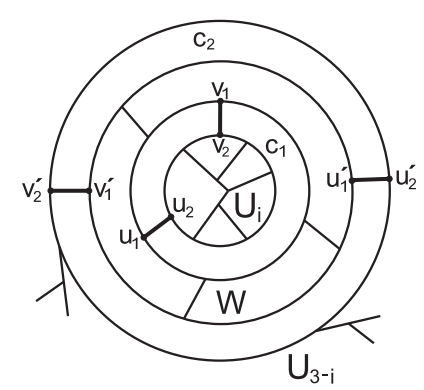

FIGURE 3.13 .

Case 3) $q=1$. If $\left|E\left(G_{2}^{\prime}\right) \cap R\right| \geq l$ or $\left|E\left(G_{1}^{\prime}\right) \cap R\right| \geq k$, the assertion follows. Suppose that $\left|E\left(G_{2}^{\prime}\right) \cap R\right|=l-1$ and $\left|E\left(G_{1}^{\prime}\right) \cap R\right|=k-1$. By the same arguments as before we conclude that in this case both the graphs $G_{1}^{\prime}-R$ and $G_{2}^{\prime}-R$ are connected. Let $g$ be a planar embedding of the connected graph $H-R$. The embedding $g$ induces planar embeddings $g_{1}$ and $g_{2}$ of the subgraphs $G_{1}^{\prime}-R$ and $G_{1}^{\prime}-R$, respectively. Since $G_{1}^{\prime}-R$ is connected, the plane subgraph $D_{2}=\left(G_{2}^{\prime} \cup B\right)-R$ is contained in a face $\gamma$ of the plane graph $G_{1}^{\prime}-R$. This means that three vertices of $G_{1}^{\prime}-R$ from the set $\left\{u_{1}, u_{1}^{\prime}, v_{1}, v_{1}^{\prime}\right\}$ are situated in the same facial circuit $c$, the circuit 
that bounds the face $\gamma$. Therefore we can draw the edge $e_{1}$ or the edge $f_{1}$ in the face $\gamma$ which gives in planar embedding of the subgraph $G_{1}-\left(f_{1} \cup R\right)$ $\left(G_{1}-\left(e_{1} \cup R\right)\right.$, respectively). Since the edges $e_{1}$ and $f_{1}$ are inessential in $G_{1}$ we have $\left|E\left(G_{1}^{\prime}\right) \cap R\right| \geq k$, contradicting to our assumption.

We conclude that in any case $|R| \geq k+l$. We thus have proved that $e d(H) \geq k+l$.

Theorem 3.14 can be used in constructing 3-connected and even cyclically 4-edge connected cubic graphs $G$ of order $16 n$ such that ed $(G) \geq 3 n$ for any natural number $n>0$. We can start from the graph $H^{\prime}$ as in Example 3.10. Let $e^{\prime}$ and $e^{\prime \prime}$ be two parallel edges in $H^{\prime}$ obtained by doubling an edge $e$ in the Heawood graph $H$. As was noted before, $H$ is cyclically 4-connected and $\zeta\left(H^{\prime}\right)=4$. Taking two copies of $H^{\prime}$, the graphs $G^{\prime}$ and $G_{1}$, and applying to them the operation of double connected sum (just as it was described in Theorem 3.14), we shall obtain a 3-connected cubic graph $H_{2}$ of order 32 . By Theorem 3.14, since $H^{\prime}$ has the property $P$, we get that $e d\left(H_{2}\right)=6$. It is not difficult to check that $\zeta\left(H_{2}\right)=4$. The thorough analysis of the proof of Theorem 3.14 shows that each bridge edge $h_{i}$ in $H_{2}$ is also inessential.

Now we can take three copies of the cubic graph $H^{\prime}$, the graphs $U_{1}, U_{2}$ and $U_{3}$ with the pairs of distinguished edges $e_{1}, f_{1}$, and $e_{2}, f_{2}$ and $e_{3}, f_{3}$, respectively, remove all them and join the resulting graphs $G_{1}, G_{2}$ and $G_{3}$ by six bridge edges $h_{i}$ as shown in Figure 3.14. The resulting cubic graph $H_{3}$ is 3 -connected and we have $e d\left(H_{3}\right)=9$. The proof of this assertion actually follows from the proof of Theorem 3.14 and uses in an essential way the facts that $H^{\prime}$ is cyclically 4 -edge connected and $H$ has the property $P$. We omit here the details.

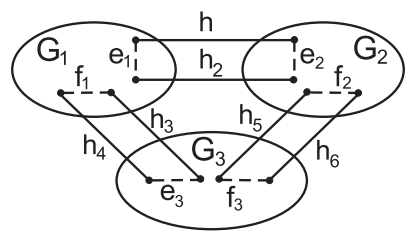

Figure 3.14. The cubic graph $H_{3}$

Iterating the process of joining the several copies of the graph $H^{\prime}$ in a cycle in the way as before, we obtain a sequence of cyclically 4-edge connected cubic graphs $H_{n}$ of order $16 n$ with $\operatorname{ed}\left(H_{n}\right)=3 n$.

Corollary 3.15. If $H$ is an $3 l$-edge deletion minimal graph in the class of 3-connected cubic graphs, then $|H| \leq 16 l$. 
Question. We provided some upper bounds for the order of minimal edge deletion (cubic) graphs and minimal genus (cubic) graphs. What about nontrivial lower bounds for these graph parameters?

Acknowledgements. The author would like to thank the referee for useful remarks and comments and for correcting some mistakes in the previous version of the manuscript.

\section{REFERENCES}

[1] Joseph Battle, Frank Harary, Yukihiro Kodama, J. W. T. Youngs. Additivity of the genus of a graph. Bull. Amer. Math. Soc., 68:565-568, 1962.

[2] Daniel Bienstock, Nathaniel Dean. Bounds for rectilinear crossing numbers. J. Graph Theory, 17(3):333-348, 1993.

[3] Gruia Călinescu, Cristina G Fernandes, Ulrich Finkler, Howard Karloff. A better approximation algorithm for finding planar subgraphs. J. Algorithms, 27(2):269-302, May 1998.

[4] Luerbio Faria, Celina M. Herrera de Figueiredo, Sylvain Gravier, Candido F. X. de Mendonça, Jorge Stolfi. On maximum planar induced subgraphs. Discrete Appl. Math., 154(13):1774-1782, 2006.

[5] Luerbio Faria, Celina M. Herrera de Figueiredo, Candido F. X. Mendonça. On the complexity of the approximation of nonplanarity parameters for cubic graphs. Discrete Appl. Math., 141(1-3):119-134, 2004.

[6] Jean-Luc Fouquet, Henri Thuillier. On some conjectures on cubic 3-connected graphs. Discrete Math., 80(1):41-57, 1990.

[7] M. R. Garey, D. S. Johnson. Crossing number is NP-complete. SIAM J. Algebraic Discrete Methods, 4(3):312-316, 1983.

[8] Jonathan L. Gross. Embeddings of cubic Halin graphs: genus distributions. Ars Math. Contemp., 6(1):37-56, 2013.

[9] Jonathan L. Gross, Thomas W. Tucker. Topological graph theory. Dover Publications, Inc., Mineola, NY, 2012.

[10] R. K. Guy. A combinatorial problem. Nabla (Bulletin of the Malayan Mathematical Society), 7:68-72, 1960.

[11] J. F. P. Hudson. Piecewise linear topology. University of Chicago Lecture Notes prepared with the assistance of J. L. Shaneson and J. Lees. W. A. Benjamin, Inc., New York-Amsterdam, 1969.

[12] Ed Pegg Jr, G. Exoo. Crossing number graphs. The Mathematica Journal, 11(2):161170, 2009.

[13] Gary L. Miller. An additivity theorem for the genus of a graph. J. Combin. Theory Ser. B, 43(1):25-47, 1987.

[14] Bojan Mohar, Carsten Thomassen. Graphs on surfaces. Johns Hopkins Studies in the Mathematical Sciences. Johns Hopkins University Press, Baltimore, MD, 2001.

[15] Bojan Mohar, Andrej Vodopivec. The genus of Petersen powers. J. Graph Theory, 67(1):1-8, 2011.

[16] Roman Nedela, Martin Škoviera. Atoms of cyclic connectivity in cubic graphs. Math. Slovaca, 45(5):481-499, 1995.

[17] Shengjun Pan, R. Bruce Richter. The crossing number of $K_{11}$ is 100. J. Graph Theory, 56(2):128-134, 2007.

[18] L. Plachta. On minimal genus cubic graphs. in preparation. 
On measures of nonplanarity of cubic graphs

[19] Gerhard Ringel. Map color theorem. Springer-Verlag, New York-Heidelberg, 1974. Die Grundlehren der mathematischen Wissenschaften, Band 209.

[20] László A. Székely. A successful concept for measuring non-planarity of graphs: the crossing number. Discrete Math., 276(1-3):331-352, 2004. 6th International Conference on Graph Theory.

[21] Carsten Thomassen. The graph genus problem is NP-complete. J. Algorithms, 10(4):568-576, 1989.

[22] Nicholas C. Wormald. Classifying $k$-connected cubic graphs. In Combinatorial mathematics, VI (Proc. Sixth Austral. Conf., Univ. New England, Armidale, 1978), volume 748 of Lecture Notes in Math., 199-206. Springer, Berlin, 1979.

Received: September 14, 2017, accepted: June 20, 2018.

Leonid Plachta

AGH University of Science and Technology (Al. Mickiewicza 30, 30-059 Cracow, Poland), \& Institute of Applied Problems of Mechanics and Mathematics of NAS of Ukraine (3-B Naukova St., 79060, Lviv, Ukraine)

Email: dept25@gmail.com 